\title{
CRISPR-Cas9 mediated gene deletions in lager yeast Saccharomyces pastorianus
}

\author{
Arthur R. Gorter de Vries ${ }^{\dagger}$, Philip A. de Groot ${ }^{\dagger}$, Marcel van den Broek and Jean-Marc G. Daran* ${ }^{*}$
}

\begin{abstract}
Background: The ease of use of CRISPR-Cas9 reprogramming, its high efficacy, and its multiplexing capabilities have brought this technology at the forefront of genome editing techniques. Saccharomyces pastorianus is an aneuploid interspecific hybrid of Saccharomyces cerevisiae and Saccharomyces eubayanus that has been domesticated for centuries and is used for the industrial fermentation of lager beer. For yet uncharacterised reasons, this hybrid yeast is far more resilient to genetic alteration than its ancestor S. cerevisiae.

Results: This study reports a new CRISPR-Cas9 method for accurate gene deletion in S. pastorianus. This method combined the Streptococcus pyogenes cas 9 gene expressed from either a chromosomal locus or from a mobile genetic element in combination with a plasmid-borne gRNA expression cassette. While the well-established gRNA expression system using the RNA polymerase III dependent SNR52 promoter failed, expression of a gRNA flanked with Hammerhead and Hepatitis Delta Virus ribozymes using the RNA polymerase II dependent TDH3 promoter successfully led to accurate deletion of all four alleles of the SelLV6 gene in strain CBS1483. Furthermore the expression of two ribozymeflanked gRNAs separated by a 10-bp linker in a polycistronic array successfully led to the simultaneous deletion of SeATF1 and SeATF2, genes located on two separate chromosomes. The expression of this array resulted in the precise deletion of all five and four alleles mediated by homologous recombination in the strains CBS1483 and Weihenstephan 34/70 respectively, demonstrating the multiplexing abilities of this gRNA expression design.
\end{abstract}

Conclusions: These results firmly established that CRISPR-Cas9 significantly facilitates and accelerates genome editing in S. pastorianus.

Keywords: Saccharomyces pastorianus, Brewing, CRISPR-Cas9, Genome editing, Ribozymes

\section{Background}

Lager beer is the most produced fermented beverage: in 2015 the worldwide production reached a global volume of $170 \times 10^{+9} \mathrm{~L}$. The fermentation workhorse of lager brewing is Saccharomyces pastorianus, a natural interspecific hybrid of Saccharomyces cerevisiae and Saccharomyces eubayanus $[1,2]$ whose domestication is thought to have occurred in central Europe (Bohemia, nowadays Czech republic) in the late Middle Ages. Its ability to ferment at low temperature, to flocculate and to produce a vast range of flavour compounds make $S$. pastorianus

\footnotetext{
*Correspondence: J.G.Daran@tudelft.nl

${ }^{\dagger}$ Arthur R. Gorter de Vries and Philip A. de Groot contributed equally to this work

Department of Biotechnology, Delft University of Technology, Van der Maasweg 9, 2629 HZ Delft, The Netherlands
}

well suited for the brewing process. In addition to their hybrid nature, S. pastorianus strains share a high degree of aneuploidy. While the first strain of $S$. pastorianus Weihenstephan 34/70 was sequenced in 2009 [2], the exact chromosome complement of lager yeast was revealed later with the introduction of next generation sequencing [3-6]. Within S. pastorianus genomes, chromosomes may be completely absent or present in up to five copies and chromosome copy numbers vary widely across different strains [4]. This intricate genome organisation significantly complicates functional gene analysis. Indeed, a simple gene deletion based on double crossover mediated by homologous recombination requires successive removal of all copies of the gene in both subgenomes by several rounds of transformation. In association with a low propensity to perform homologous 
recombination, the difficulty to delete high copy number genes may explain the quasi-absence of examples of functional characterisation of $S$. pastorianus genes in the scientific literature based on impact of gene deletion [7-9]. Instead a S. pastorianus gene or allele is usually cloned in S. cerevisiae and characterised based on the impact of the overexpression. However, such approaches do not take into account the role of the orthologous gene harboured by the other sub-genome, the possible occurrence of paralogs, and the gene expression regulation of the gene in its allo-aneuploid genetic background. Therefore, tools are needed to achieve efficient genome editing in allo-aneuploid S. pastorianus not only to enable targeted genetic modification, but also to enable functional gene analysis.

The exposed DNA strand ends resulting from a DNA double strand break (DSB) are extremely recombinogenic [10, 11]. Even in Saccharomyces cerevisiae that exhibits a natural inclination to perform homologous recombination, introduction of a programmed DSB by combining the insertion an I-SceI restriction site in a chromosomal locus and expression of the endonuclease encoding gene SCEI showed substantial stimulation of homologous recombination at the cut site enabling the correct assembly of multiple DNA fragments [12]. Although efficient, the use of SceI induced DSB is limited since it requires the insertion of the recognition site prior its utilisation. In the past 5 years, the advent of the CRISPR (clustered regularly interspaced short palindromic repeat)-Cas9 (CRISPR-associated protein 9) system derived from Streptococcus pyogenes has considerably transformed genome engineering approaches [13, 14]. The system comprises two elements: a short chimeric RNA that derives from the fusion of the tracr and crRNA called guide RNA (gRNA), and the endonuclease Cas9 $[13,14]$. By forming a complex with Cas9, the gRNA provides sequence specificity to the system. The heteroduplex formed by the gRNA and the genomic target places the endonuclease which generates a blunt ended DSB. The systems has been successfully implemented in S. cerevisiae [15-19], which broadened genome editing possibilities by allowing multiplexing $[15,16,18]$ and high precision in vivo site-directed mutagenesis [15]. The expression of the gRNA has been a point of attention since the gRNA secondary structures are crucial for the formation of the complex with Cas9. Therefore the $5^{\prime}$ capping and $3^{\prime}$ polyadenylation present in RNA polymerase II transcripts have to be avoided. By analogy with the expression of gRNA in human cell lines [14], placing the gRNA behind the control of a RNA polymerase III dependent promoter (e.g. SNR52p) resulted in expression of an active gRNA lacking these modifications [16]. In addition, due to the lack of polyadenylation-mediated export to the cytosol, RNA polymerase III transcribed gRNAs reside in the nucleus longer where they can form a complex with Cas9.

However gRNA expression from a RNA polymerase III was shown to result in low and unstable transcript levels [20]. To overcome this issue while avoiding inactivation of the gRNA by $5^{\prime}$ capping and $3^{\prime}$ polyadenylation, the gRNA can be flanked by two ribozymes molecules and expressed by RNA polymerase II. Upon transcription the ribozymes self-cleave, resulting in removal of $5^{\prime}$ and 3- ends and release of a mature gRNA $[19,21]$. Such CRISPR-Cas9 systems have been confirmed to mediate efficient genome editing in multiple cell types already, such as human cell lines [13, 14, 22], mice [23], zebrafish [24], Caenorhabditis elegans [25, 26], Drosophila [27], yeasts $[15,16,28,29]$, and plants [30-32].

The goal of the present study was to explore the use of CRISPR-Cas9 in S. pastorianus, a yeast with low genetic accessibility that is characterised by a unique allo-aneuploid genome. To this end, we present the construction of molecular tools to achieve efficient single and double simultaneous gene deletions. The successful application of this methodology offers an opportunity to get a deeper understanding of hybrid yeast biology.

\section{Methods}

\section{Strains and growth conditions}

The S. pastorianus and cerevisiae strains used in this study are listed in Table 1 and a construction flow-chart is provided in Fig. 1.

Under nonselective conditions, Saccharomyces pastorianus and cerevisiae strains were grown in complex medium (YPD) containing $10 \mathrm{~g} \mathrm{~L}^{-1}$ yeast extract, $20 \mathrm{~g} \mathrm{~L}^{-1}$ peptone, and $20 \mathrm{~g} \mathrm{~L}^{-1}$ glucose. For nourseothricin selection, YPD medium was supplemented with $100 \mu \mathrm{g} \mathrm{L}{ }^{-1}$ of the antibiotic. Synthetic media (SM) containing $20 \mathrm{~g} \mathrm{~L}^{-1}$ glucose, $3 \mathrm{~g} \mathrm{~L}^{-1} \mathrm{KH}_{2} \mathrm{PO}_{4}, 0.5 \mathrm{~g} \mathrm{~L}^{-1} \mathrm{MgSO}_{4} 7 \mathrm{H}_{2} \mathrm{O}, 5 \mathrm{~g} \mathrm{~L}^{-1}$ $\left(\mathrm{NH}_{4}\right)_{2} \mathrm{SO}_{4}, 1 \mathrm{~mL} \mathrm{~L}^{-1}$ of a trace element solution and of a vitamin solution was prepared as previously described [33]. For selection of yeast strains harboring an acetamidase marker [34] $\left(\mathrm{NH}_{4}\right)_{2} \mathrm{SO}_{4}$ was replaced by $0.6 \mathrm{~g} \mathrm{~L}^{-1}$ acetamide as nitrogen source and $6.6 \mathrm{~g} \mathrm{~L}^{-1} \mathrm{~K}_{2} \mathrm{SO}_{4}$ to compensate for sulfate (SM-Ac). Loss of the acetamide marker was selected for on SM containing $2.3 \mathrm{~g} \mathrm{~L}^{-1}$ fluoroacetamide (SM-Fac) [34]. The $\mathrm{pH}$ in all media was adjusted to 6.0 with $\mathrm{KOH}$. Solid media were prepared by adding $2 \%$ agar to the various media. The strains of S. pastorianus and cerevisiae were incubated at 20 and $30{ }^{\circ} \mathrm{C}$ respectively.

Shake flask cultures of $S$. pastorianus were grown at $20{ }^{\circ} \mathrm{C}$ in $500 \mathrm{~mL}$ flasks containing $100 \mathrm{~mL}$ complete medium (YPD) with $20 \mathrm{~g} \mathrm{~L}^{-1}$ glucose in an Innova 43/43R shaker (Eppendorf, Hauppauge, NY) set at 
Table 1 Strains used throughout this study

\begin{tabular}{|c|c|c|c|}
\hline Name & Species & Genotype & Source \\
\hline CBS1483 & S. pastorianus & Wildtype & {$[4]$} \\
\hline $\operatorname{IMX} 1187$ & S. pastorianus & SPR3::AaTEF1p-Spcas9 $9^{\text {147YP411T }}$-SCPHO5t & This study \\
\hline IMX1205 & S. pastorianus & SPR3::AaTEF1p-Spcas9 $9^{D 147 Y P 411 T}$-ScPHO5t $\triangle$ Seilv6 & This study \\
\hline IMK771 & S. pastorianus & $\Delta$ Seilv6 & This study \\
\hline IMK786 & S. pastorianus & $\Delta$ Seatf1 $\Delta$ Seatf2 & This study \\
\hline Weihenstephan 34/70 & S. pastorianus & Wildtype & {$[2,66]$} \\
\hline IMK813 & S.pastorianus & $\Delta$ Seatf1 $\Delta$ Seatf2 & This study \\
\hline CEN.PK113-7D & S. cerevisiae & MATa MAL2-8C & {$[67]$} \\
\hline IMX585 & S. cerevisiae & 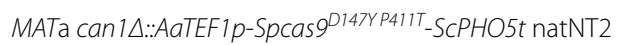 & {$[15]$} \\
\hline
\end{tabular}

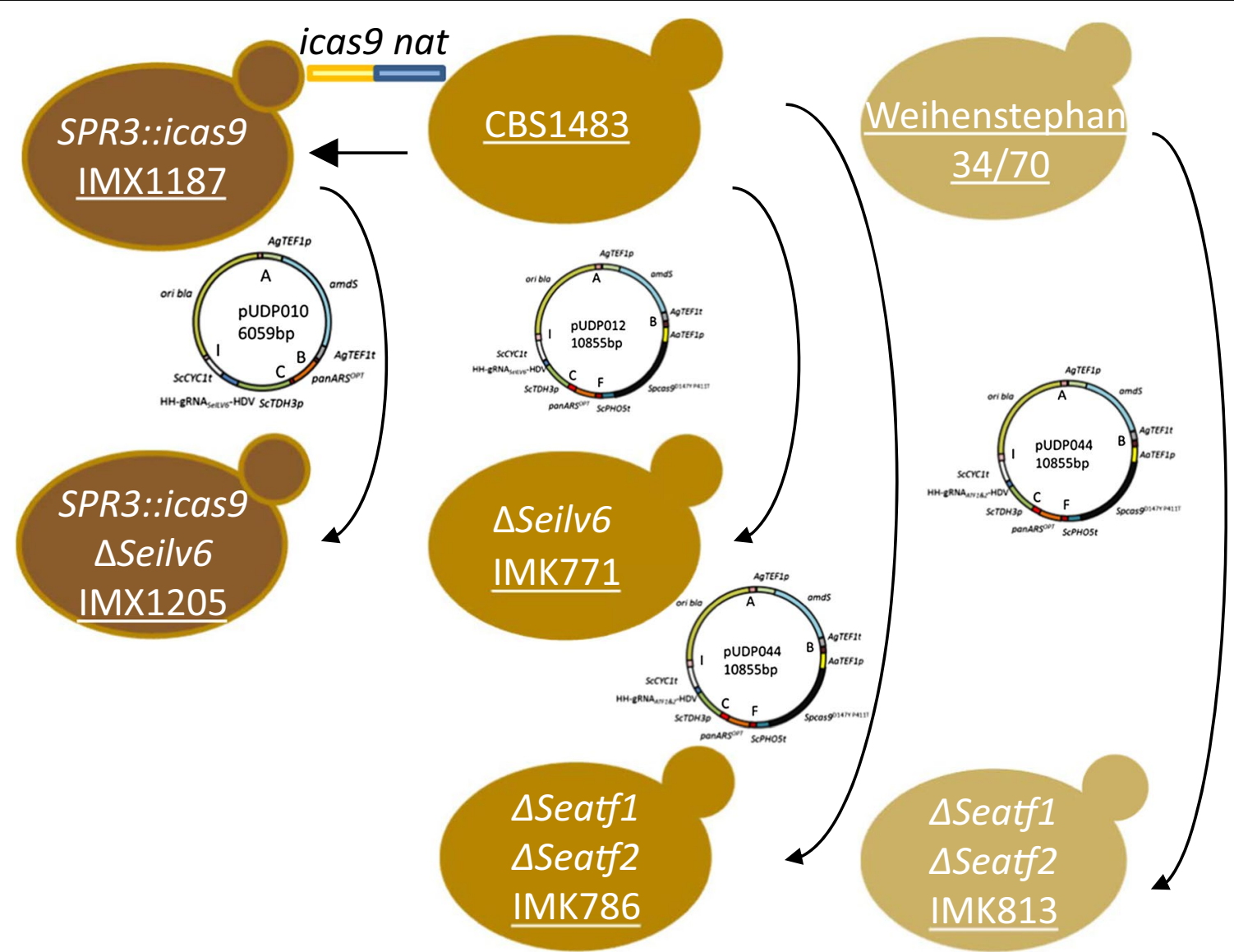

Fig. 1 Strains construction flow-chart. Schematic representation of the different strain lineages constructed in this study. The strain name is underlined and each arrow indicates a transformation step

$200 \mathrm{rpm}$. Frozen stocks were prepared by addition of glycerol $(30 \% \mathrm{v} / \mathrm{v})$ to exponentially growing shake-flask cultures of S. cerevisiae, S. pastorianus and overnight cultures of Escherichia coli and stored aseptically in $1 \mathrm{~mL}$ aliquots at $-80^{\circ} \mathrm{C}$.
For growth studies in shake flasks, S. pastorianus strains were grown in shake flasks with complete medium YPD. Growth rates were based on optical density at $660 \mathrm{~nm}\left(\mathrm{OD}_{660}\right)$ measurements using a Libra S11 spectrophotometer (Biochrom, Cambridge, United 
Kingdom). Specific growth rates were calculated from exponential fits of the $\mathrm{OD}_{660}$ against time.

\section{Plasmid construction}

All plasmids and primers used during this study are shown in Tables 2 and 3, respectively. The DNA parts harboured by the plasmids pUD527, pUD528, pUD530, pUD531, pUD532 pUD536 and pUD573 were de novo synthesised at GeneArt (Thermo Fisher Scientific, Waltham, MA). Unless specified, plasmids were propagated and stored in E. coli strain XL1-blue. Yeast transformation was done by electroporation using $50 \mu \mathrm{L}$ of competent cells and up to $5 \mu \mathrm{L}$ DNA as previously described [35] and transformed cells were incubated in $0.5 \mathrm{~mL}$ YPD during $1 \mathrm{~h}$, after which they were re-suspended in $100 \mu \mathrm{L}$ of sterile demi-water and plated on selective medium. High fidelity PCR amplification was performed using Phusion polymerase (Thermo Fisher Scientific) according to supplier's instructions.

pUD423 was assembled from plasmids pCT, pUD528 and pUC19. The Streptococcus pyogenes cas 9 open reading frame (cas $9^{\mathrm{D} 147 \mathrm{Y}}$ P411T $\left.[36]\right)$ was amplified from the plasmid pCT (Addgene plasmid \#60621) (https://www. addgene.org/) using the primers 9390 and 9391. The AaTEF1 promoter flanked upstream by short homology flank (SHF) B was amplified from the plasmid pUD528 using the primers 3841 and 9394 . The ScPHO5 terminator fragment was amplified from pUD528 using the primers 9392 and 9393, resulting in the addition of SHR F downstream of the terminator. The three fragments together with the pUC19 backbone [37] amplified with the primers 7389 and 9395 were assembled in vitro using ligase chain reaction (LCR) with primers 9396-9399 as bridging oligonucleotides as described previously [38] and the resulting plasmid pUD423 was verified using digestion with $N d e$ I.

The cassette for integration of cas 9 into the SPR3 locus was assembled on pUD526. Flanks for homologous recombination of about 500 bp were amplified from genomic DNA of CBS1483 using primers sets 10432/10433 and 10434/10435 adding NotI restriction sites upstream of the left homology arm and downstream of the right homology arm and 40 bp homology flanks on both sides of the homology arms for "Gibson" assembly [39]. The cas 9 expression cassette was amplified from plasmid pUD423 using primers 10426 and 10427, the nourseothricin marker was amplified from pMEL15 [15] using primers 3597 and 10436 adding a 40 bp homology flank upstream of the nat gene, and the plasmid backbone was amplified from plasmid pUC19 using primers 7389

Table 2 Plasmids used throughout the study

\begin{tabular}{|c|c|c|c|}
\hline Name & Relevant genotype & Source & Addgene ID\# \\
\hline $\mathrm{pCT}$ & ori amp ${ }^{r}$ ARS4 CEN6 LEU2 AaTEF1p-Spcas9 $9^{D 147 Y \text { P411T }}$-SCPHO5 & {$[36]$} & \\
\hline PMEL15 & ori amp 2 $2 \mu \mathrm{m}$ natNT2 SNR52p-gRNA CAN1-SUP4t & [15] & \\
\hline pROS12 & ori amp $2 \mu \mathrm{m}$ hphNT1 gRNA ${ }_{\text {CAN1 }}$ gRNA ${ }_{\text {ADE2 }}$ & [15] & \\
\hline pUC19 & ori amp ${ }^{r}$ LacZ multiple cloning site & {$[68]$} & \\
\hline pUD423 & ori amp ${ }^{r}$ AaTEF1p-Spcas9 ${ }^{D 147 Y P 411 T}$-SCPHO5t & GeneArt $^{\mathrm{TM}}$ & \\
\hline pUD526 & ori amp ${ }^{r \text { Notl }}$ HRL AaTEF1p-Spcas9 $9^{\text {147YP411T }}$-ScPHO5t natNT2 HRM ${ }^{\text {Notl }}$ & GeneArt $^{\mathrm{TM}}$ & \\
\hline pUD527 & ori kan ${ }^{r}$ SHRA AgTEF1p-amdS-AgTEF1t SHRB & GeneArt $^{\mathrm{TM}}$ & \\
\hline pUD528 & ori kan' SHRB AaTEF1p-Spcas9 $9^{\text {D147Y P411T }}$-PHO5t & GeneArt $^{\mathrm{TM}}$ & \\
\hline pUD530 & ori kan ${ }^{r}$ SHRB panARSopt SHRC & GeneArt $^{\mathrm{TM}}$ & \\
\hline pUD531 & ori kan ${ }^{r}$ SHRC TDH3p ${ }^{\text {Bsal Bsal }}$ CYC1t SHRI & GeneArt $^{\mathrm{TM}}$ & \\
\hline pUD532 & ori kan' SHRI bla ori SHRA & GeneArt $^{\mathrm{TM}}$ & \\
\hline pUD536 & ori amp ${ }^{r \text { Bsal }}$ gRNA $A_{\text {Sellvg }}^{\text {Bsal }}$ & GeneArt $^{\mathrm{TM}}$ & \\
\hline pUD573 & ori $a m p^{r \text { Bsal }}$ gRNA $_{\text {SeATF } 1}$ gRNA $_{\text {SEATF2 }}^{\text {sal }}$ & GeneArt $^{\mathrm{TM}}$ & \\
\hline pUDP003 & ori amp ${ }^{r}$ panARSopt AgTEF1p-amdS-AgTEF1t TDH3p Bsal Bsal CYC1t & This study & 101164 \\
\hline pUDP004 & 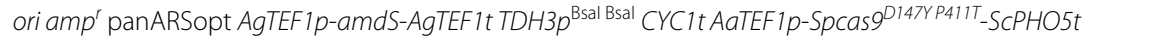 & This study & 101165 \\
\hline pUDP010 & ori amp panARSopt AgTEF1p-amdS-AgTEF1t TDH3p-HH-gRNA sellv6 $_{\text {-HDV-CYC1t }}$ & This study & 101166 \\
\hline pUDP012 & $\begin{array}{l}\text { ori amp panARSopt AgTEF1p-amdS-AgTEF1t TDH3p-HH-gRNA } \text { Sellv6 }_{\text {-HDV-CYC1t AaTEF1p-Spcas } 9 \text { D147Y P411T_ }} \\
\text { SCPHO5t }\end{array}$ & This study & 101167 \\
\hline pUDP044 & 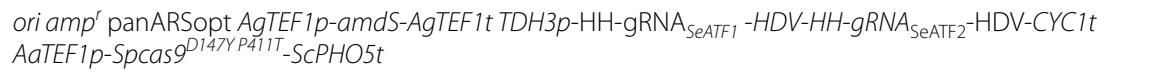 & This study & 101168 \\
\hline pUDR107 & ori amp $2 \mu \mathrm{m}$ hphNT1 gRNA URA3 & This study & \\
\hline
\end{tabular}

HRL and HRM indicate the left and right homology arms for integration on the SPR3 locus, SHR stands for synthetic homologous recombination sequence and enzyme digestion sites are indicated in superscript

a https://www.addgene.org/ 
Table 3 Primers used in this study

\begin{tabular}{|c|c|c|}
\hline Name & Sequence & Purpose \\
\hline 3274 & TATTCACGTAGACGGATAGGTATAGC & Amplification SHRI \\
\hline 3275 & GTGCCTATTGATGATCTGGCGGAATG & Amplification SHR A \\
\hline 3276 & GTTGAACATTCTTAGGCTGGTCGAATC & Amplification SHR B \\
\hline 3283 & ACGTCTCACGGATCGTATATGC & Amplification SHRC \\
\hline 3597 & ATTAAGGGTTCTCGAGAGC & Amplification natNT2 \\
\hline 3750 & GAGGCGTTAGTTTGGCTAATGAG & Diagnostic primer \\
\hline 3841 & CACCTTTCGAGAGGACGATG & Amplification SHR B \\
\hline 3847 & ACTATATGTGAAGGCATGGCTATGG & Amplification SHR A \\
\hline 3856 & CTAGCGTGTCCTCGCATAGTTC & Amplification SHRC \\
\hline 4068 & GCCTACGGTTCCCGAAGTATGC & Amplification SHR I \\
\hline 6005 & GATCATTTATCTTTCACTGCGGAGAAG & pROS12 backbone \\
\hline 7389 & GGTTTCTTAGACGTCAGGTGGC & pUC19 backbone \\
\hline 8076 & $\begin{array}{l}\text { GTTTAGCTCTATGGTGCAAAATTCTCCAGAAAAAAGGGATCCATAGAAAAGAATATG } \\
\text { TCTAATTGAAAAATAGATATGTACCATAAGTAAAGTGCATGCGTGTATACCGAAACC } \\
\text { AAGACA }\end{array}$ & Repair DNA SeURA3 \\
\hline 8077 & $\begin{array}{l}\text { TGTCTTGGTTTCGGTATACACGCATGCACTTTACTTATGGTACATATCTATTTTTCAATT } \\
\text { AGACATATTCTTTTCTATGGATCCCTTTTTTCTGGAGAATTTTGCACCATAGAGCT } \\
\text { AAAC }\end{array}$ & Repair DNA SeURA3 \\
\hline 8314 & $\begin{array}{l}\text { TGCGCATGTTTCGGCGTTCGAAACTTCTCCGCAGTGAAAGATAAATGATCTTGACTG } \\
\text { ATTTTTCCATGGAGTTTTAGAGCTAGAAATAGCAAGTTAAAATAAG }\end{array}$ & pROS12 + SCURA3 gRNA \\
\hline 8553 & $\begin{array}{l}\text { TGCCCAGTATTCTTAACCCAACTGCACAGAACAAAAACCTGCAGGAAACGAAGA } \\
\text { TAAATCAAAACTGTATTATAAGTAAATGCATGTATACTAAACTCACAAATTAGAGC } \\
\text { TTCAATTTAA }\end{array}$ & Repair DNA ScURA3 \\
\hline 8554 & $\begin{array}{l}\text { TTAAATTGAAGCTCTAATTTGTGAGTTTAGTATACATGCATTTACTTATAATACAGTT } \\
\text { TTGATTTATCTTCGTTTCCTGCAGGTTTTTGTTCTGTGCAGTTGGGTTAAGAA } \\
\text { TACTGGGCA }\end{array}$ & Repair DNA ScURA3 \\
\hline 9310 & TCGCCTGCAAATCGTCATCG & Diagnostic primer ILV6 \\
\hline 9311 & CCTTAGAAACATCCGAGCTCCTCCTGGGCCTCTATACATC & Repair fragment construction \\
\hline 9312 & GATGTATAGAGGCCCAGGAGGAGCTCGGATGTTTCTAAGG & Repair fragment construction \\
\hline 9313 & AGCTGGTCGCCAAGGACTAC & Diagnostic primer ILV6 \\
\hline 9314 & CTACTGCGCCAATTGATGAC & Diagnostic primer ScURA3 \\
\hline 9317 & GCCCTACACGTTCGCTATGC & Diagnostic primer ScURA3 \\
\hline 9318 & GTTGACACAGTCCGTGAAAC & Diagnostic primer SeURA3 \\
\hline 9321 & GGCGCATTGGAGTCAATGAG & Diagnostic primer SeURA3 \\
\hline 9390 & ATGGATTATAAAGATGACGATGACAAAC & Amplification cas 9 \\
\hline 9391 & CCGCTCAGACCTTTCTCTTC & Amplification cas9 \\
\hline 9392 & TTTTGTATAACTAAATAATATTGGAAACTAAATACG & Amplification ScPHO5t \\
\hline 9393 & $\begin{array}{l}\text { TGCCGAACTTTCCCTGTATGAAGCGATCTGACCAATCCTTTGCCGTAGTTTCA } \\
\text { ACGTATGTTTTCATTTTTGCGATGCCAG }\end{array}$ & Amplification ScPHO5t + addition SHR F \\
\hline 9394 & TGTTGATTATGTTTTTAAGAACTACTCAGAATG & Amplification AaTEF1p \\
\hline 9395 & AGGCCAGGAACCGTAAAAAG & pUC19 backbone \\
\hline 9396 & $\begin{array}{l}\text { ATTTCATTCTGAGTAGTTCTTAAAAACATAATCAACAATGGATTATAAAGATGACGAT } \\
\text { GACAAACCTCCAAAAA }\end{array}$ & LCR bridging oligo \\
\hline 9397 & $\begin{array}{l}\text { TGACCCTCCAAAAAAGAAGAGAAAGGTCTGAGCGGTTTTGTATAACTAAATAATAT } \\
\text { TGGAAACTAAATAC }\end{array}$ & LCR bridging oligo \\
\hline 9398 & TTCATACAGGGAAAGTTCGGCAGGTTTCTTAGACGTCAGGTGGC & LCR bridging oligo \\
\hline 9399 & CCTTTTTACGGTTCCTGGCCTCACCTTTCGAGAGGACGATG & LCR bridging oligo \\
\hline 9663 & $\begin{array}{l}\text { CATACGTTGAAACTACGGCAAAGGATTGGTCAGATCGCTTCATACAGGGAA } \\
\text { AGTTCGGCATCAACATCTTTGGATAATATCAGAATGAG }\end{array}$ & Amplification panARSopt + addition of SHR F \\
\hline 10426 & AATCTATAATCAGTCCATAGTCAACAAGAGCC & Amplification AaTEF1p \\
\hline 10427 & TTTTCATTTTTGCGATGCCAGTTCTTTG & Amplification ScPHO5t \\
\hline 10432 & $\begin{array}{l}\text { AAAAACGCCAGCAACGCGGCCTTTTTACGGTTCCTGGCCTGCGGCCGCGCTTCCA } \\
\text { GGTTTGGCACTGTC }\end{array}$ & 40 bp to pUC19 + Notl restriction + Left Homology arm fw \\
\hline
\end{tabular}


Table 3 continued

\begin{tabular}{|c|c|c|}
\hline Name & Sequence & Purpose \\
\hline 10433 & $\begin{array}{l}\text { ACTTTGAGGGCTCTTGTTGACTATGGACTGATTATAGATTTACGAAGGCACTTTGC } \\
\text { ATGGG }\end{array}$ & Left Homology arm rv + 40 bp to AaTEF1p \\
\hline 10434 & $\begin{array}{l}\text { GACAACACCTGTTGTAATCGAGCTCTCGAGAACCCTTAATGCGCGACATCAAATACC } \\
\text { TTTGTCC }\end{array}$ & 40 bp to natNT2 + right homology arm fw \\
\hline 10435 & $\begin{array}{l}\text { CACATTTCCCCGAAAAGTGCCACCTGACGTCTAAGAAACCGCGGCCGCACGA } \\
\text { CGCGGACGAAGAGAAAG }\end{array}$ & Right homology arm rv + Notl restriction $+40 \mathrm{bp}$ to pUC19 \\
\hline 10436 & $\begin{array}{l}\text { ATAAGGGAAACTCAAAGAACTGGCATCGCAAAAATGAAAATAGGTCTAGAGATCTG } \\
\text { TTTAGC }\end{array}$ & $40 \mathrm{bp}$ to $\mathrm{ScPHO} 5 \mathrm{t}+$ natNT2 fw \\
\hline 10686 & $\begin{array}{l}\text { GAGTAAAGAAGCTCATCATTTATATAGATACGTTATGTAGATGTATAGAGGCCCAGGAG } \\
\text { GGAGCTCGGATGTTTCTAAGGCTCTGTATGTACAAACTACGTATGTGACTTATACAT } \\
\text { TGCT }\end{array}$ & Repair DNA ILV6 \\
\hline 10687 & $\begin{array}{l}\text { AGCAATGTATAAGTCACATACGTAGTTTGTACATACAGAGCCTTAGAAACATCCGA } \\
\text { GCTCCCTCCTGGGCCTCTATACATCTACATAACGTATCTATATAAATGATGAGC } \\
\text { TTCTTTACTC }\end{array}$ & Repair DNA ILV6 \\
\hline 10992 & $\begin{array}{l}\text { GTTCAAGATGAATGTCTTGTCAAGATGATACAGAATGGGCATTCCCGGCGTATGGGAT } \\
\text { CTTCATGGCATCAAGCTTTTTCATTGGGTGTTTCTTCGACTAATGTGAAGGGAATGA } \\
\text { ACATT }\end{array}$ & Repair DNA SeATF1 \\
\hline 10993 & $\begin{array}{l}\text { AATGTTCATTCCCTTCACATTAGTCGAAGAAACACCCAATGAAAAAGCTTGATGCCAT } \\
\text { GAAGATCCCATACGCCGGGAATGCCCATTCTGTATCATCTTGACAAGACATTC } \\
\text { ATCTTGAAC }\end{array}$ & Repair DNA SeATF1 \\
\hline 10994 & $\begin{array}{l}\text { TTTGCTGTTTTGCGTAGGCAAAACATGTATTCGAATTTCGCTGTTTATGGGGAACTGA } \\
\text { ATAACGTTGGTGGTATGAACATGGACATGAGCGTAGTTCAGGGCACTCTACGGAA } \\
\text { TCGGGGC }\end{array}$ & Repair DNA SeATF2 \\
\hline 10995 & $\begin{array}{l}\text { GCCCCGATTCCGTAGAGTGCCCTGAACTACGCTCATGTCCATGTTCATACCACCAACG } \\
\text { TTATTCAGTTCCCCATAAACAGCGAAATTCGAATACATGTTTTGCCTACGCAAAAC } \\
\text { AGCAAA }\end{array}$ & Repair DNA SeATF2 \\
\hline 10996 & ATGAGAAAAATCAGGCCCCC & Diagnostic primer \\
\hline 10997 & CTAAGGGCCTAAAAGGAGAGC & Diagnostic primer \\
\hline 10998 & GAAGGATACGAACCACATATCACG & Diagnostic primer \\
\hline 10999 & TAAAGCGACGCAAATTCGCC & Diagnostic primer \\
\hline 11000 & CAGAAGAAAGCCAATTTAGCAG & Diagnostic primer \\
\hline 11001 & TCAGGGATTTAAAAGCAGAGC & Diagnostic primer \\
\hline 11002 & GGATAGTTTAGAGGAATACGAACCG & Diagnostic primer \\
\hline 11003 & TATACGAGACCCGCGACG & Diagnostic primer \\
\hline
\end{tabular}

SHR sequences are shown in bold, gRNA sequences are shown in italics and digestion enzyme recognition sites are underlined

and 9395. Next, 0.2 pmol of each fragment were assembled into pUD526 using NEBuilder ${ }^{\circledR}$ HiFi DNA Assembly Master Mix (New England BioLabs, Ipswich, MA), verified by digestion with BamHI and NotI. The integration cassette was obtained by digestion of the plasmid using NotI followed by gel purification.

pUDP003 was assembled from plasmids pUD527, pUD530, pUD531 and pUD532 (Fig. 2). The amdS selection cassette [34] was amplified from pUD527 using primers 3847 and 3276 containing SHF A and B flanks. The synthetic pangenomic yeast replication origin panARSopt [40] was amplified from pUD530 using primers 3841 and 3856 containing SHF B and C flanks. The gRNA introduction site was amplified from pUD531 using primer 3283 and 4068 containing SHF C and I flanks. The $E$. coli replication origin from pBR322 and the bla gene conferring resistance to $\beta$-lactam antibiotics were amplified from pUDP532 using primers 3274 and
3275 containing SHF I and A flanks. The amplified fragments were digested with DpnI, gel purified and quantified using a NanoDrop 2000 spectrophotometer (Thermo Fisher Scientific). $0.2 \mathrm{pmol}$ of each fragment were assembled into pUDP003 using NEBuilder ${ }^{\circledR} \mathrm{HiFi}$ DNA Assembly Master Mix (New England BioLabs). The resulting plasmid pUDP003 was verified by restriction analysis using SspI.

pUDP004 was assembled from plasmids pUD423, pUD527, pUD530, pUD531 and pUD532 (Fig. 2). The amdS selection cassette [34] was amplified from pUD527 using primers 3847 and 3276 introducing SHF A and B flanks. The cas 9 expression cassette was amplified from pUD423 using primers 3841 and 9393 containing SHF B and $F$ flanks. The synthetic pangenomic yeast replication origin panARSopt [40] was amplified from pUD530 using primers 9663 and 3856 containing the SHF C flank and introducing the SHF F flank, thereby replacing the SHF 


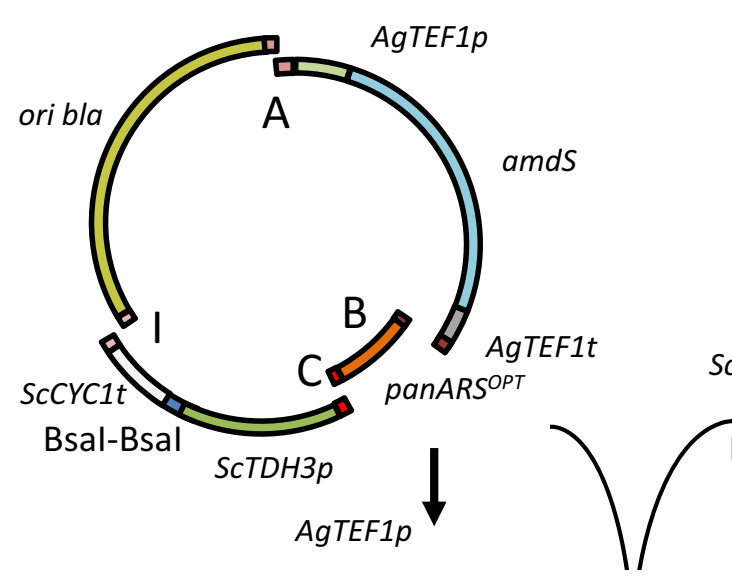

In vitro

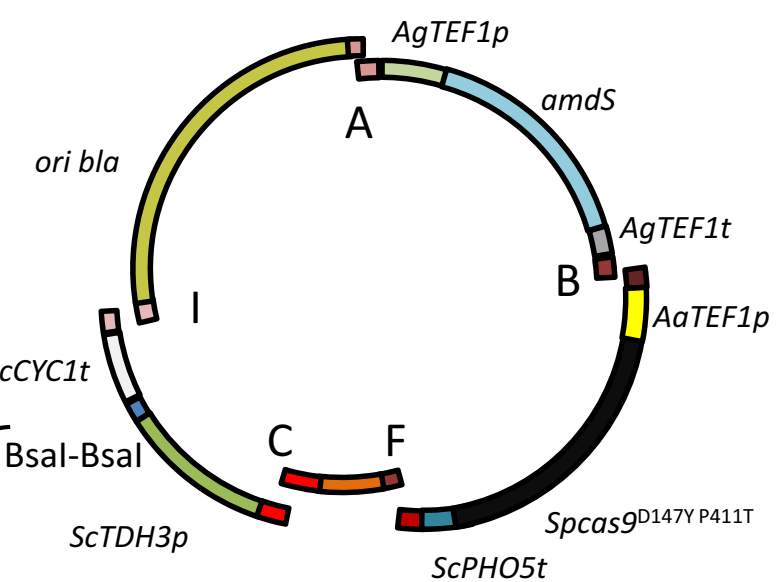

panARSOPT

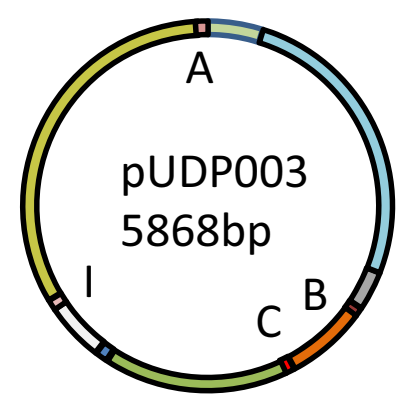

"Gibson" assembly


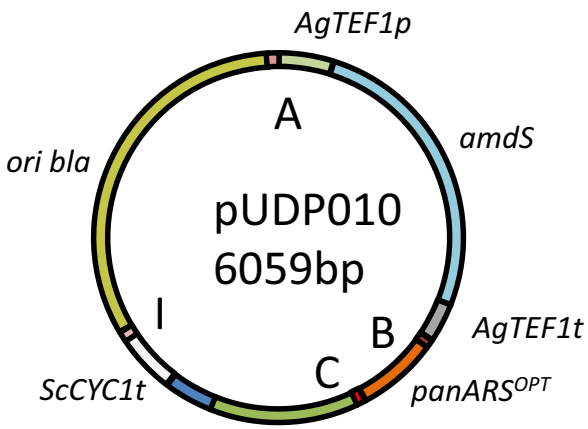

$\mathrm{HH}-g R N A_{\text {SelLV6 }}-\mathrm{HDV}$ ScTDH3p

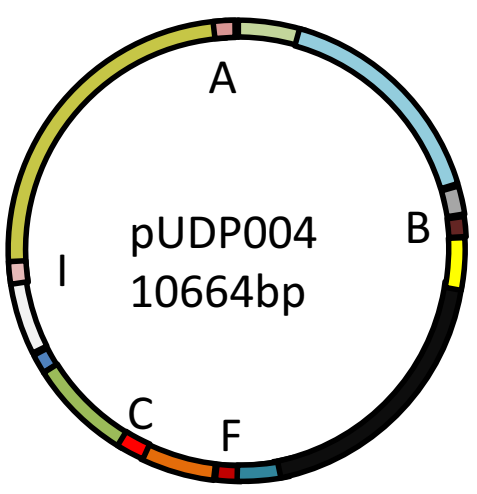

DNA ligase T4

Fig. 2 Construction of the gRNA expression plasmids pUDP003 and pUDP004. In vitro "Gibson" assembly [39] of functional parts containing an amdS selection marker cassette, a synthetic pangenomic yeast replication origin panARSopt, an E. coli replication origin from pBR322 and the bla gene conferring resistance to $\beta$-lactam antibiotics and a gRNA expression cassette using 60 bp synthetic homologous recombination sequences into pUDP003 and with the addition of a fragment carrying a Spcas9 expression part into pUDP004. The ribozymes flanked gRNA is next directionally inserted into pUDP003 or pUDP004 using Bsal digestion and ligation yielding the gRNA expressing plasmids pUDP010 and pUDP012 respectively

B flank. The gRNA introduction site was amplified from pUD531 using primers 3283 and 4068 containing SHF C and I flanks. The E. coli replication origin from pBR322 and the bla gene conferring resistance to $\beta$-lactam antibiotics were amplified from pUDP532 using primers 3274 and 3275 containing SHF I and A flanks. The amplified 
fragments were digested with DpnI, gel purified and quantified using a NanoDrop 2000 spectrophotometer (ThermoFischer Scientific). $0.2 \mathrm{pmol}$ of each fragment were assembled into pUDP004 using NEBuilder ${ }^{\circledR}$ HiFi DNA Assembly Master Mix (New England BioLabs). The assembled plasmid pUDP004 was verified by restriction analysis using PdmI.

The gRNA sequences for pUDP type plasmids were designed such that they could be synthesized and inserted into pUDP003 or pUDP004 by digestion with BsaI and ligation. From $5^{\prime}$ to $3^{\prime}$, the sequences were composed of a BsaI recognition site yielding correct sticky ends "GGTCTCGCAAA", followed by the hammerhead ribozyme with the first six nucleotides being the reverse complement $\left(^{\mathrm{C}}\right)$ of the first six nucleotides of the gRNA spacer " ${ }^{c}{ }_{6}^{c}{ }_{5}^{c} N_{4}^{c} N_{3}^{c} N_{2}^{c} N_{1}$ CUGAUGAGUCCGUGAGGACGAAACGAGUAAGC UCGUC", followed by the 20 nucleotide gRNA spacer designed as previously [15], followed by the structural gRNA "GUUUUAGAGCUAGAAAUAGCAAGUUAAAAUAA GGCUAGUCCGUUAUCAACUUGAAAAAGUGGC ACCGAGUCGGUGCUUUU", followed by the Hepatitis Delta Virus ribozyme "GGCCGGCAUGGUCCCAGCC UCCUCGCUGGCGCCGGCUGGGCAACAUGCUU CGGCAUGGCGAAUGGGAC", followed again by a BsaI recognition site yielding correct sticky ends "ACAGCGAGACC". For multiplexing, linker "ACAGCGCAAA" was added between the HDV ribozyme of the first gRNA and the HH ribozyme of the second gRNA. Plasmids pUD536, containing the gRNA sequence targeting SeILV6, and pUD573, containing a polycistronic array with gRNAs targeting SeATF1 and SeATF2, were de novo synthesised at GeneArt (Thermo Fisher Scientific). The plasmid pUDP010, expressing gRNA $_{\text {SelLV6 }}$, was constructed in a one-pot reaction by digesting pUDP003 and pUD536 using BsaI and ligating with T4 ligase. Similarly pUDP012, expressing gRNA SeILV6 $_{6}$ and Spcas $9^{\text {D147Y P411T }}$, was assembled from pUDP004 and pUD536 and pUDP044, expressing gRNA $_{\text {SeATF1 } 1: \mathrm{gRNA}_{\text {SeATF2 }} \text { and }}$ Spcas $9^{\mathrm{D} 147 \mathrm{Y}} \mathrm{P} 41 \mathrm{~T} \mathrm{~T}$ was assembled from pUDP004 and pUD573. Correct assembly of pUDP010 was verified by restriction analysis with SspI and correct assembly of pUDP012 and pUDP044 was verified by restriction analysis using PdmI. Plasmid pUDR107, expressing gRNA $_{U R A 3}$, was constructed using NEBuilder ${ }^{\circledR} \mathrm{HiFi}$ DNA Assembly Master Mix by assembling the $2 \mu \mathrm{m}$ fragment amplified from pROS12 with primer 8314 and the plasmid backbone amplified from pROS12 with primer 6005 as previously described in [15].

Plasmids pUDP003, pUDP004, pUDP010, pUDP012 and pUDP044 were deposited at addgene (http://www. addgene.org/) (Table 1).

\section{Strain construction}

The strain IMX1187 was constructed by transforming CBS1483 with $1 \mu \mathrm{g}$ of the NotI-digested and gel purified integration cassette from pUD526 by electroporation and plated on YPD with nourseothricin (Fig. 1). After 5 days, 14 colonies had grown and integration of cas 9 was confirmed using primers 3750 and 9394. One of the colonies was stocked and sequenced.

IMX1205 (Fig. 1) was constructed by transforming IMX1187 by electroporation with $500 \mathrm{ng}$ of pUDP010 and $1 \mu \mathrm{g}$ of a $120 \mathrm{bp}$ repair fragment obtained by mixing an equimolar amount of primers 10686 and 10687. Transformants were selected on SM-Ac plates. Transformants were confirmed using primers 9310 and 9313 . Prior stocking the isolate was successively streaked out on SM-Ac, YPD and SM-FAc plates. Genotype was systematically verified after each plating round with primers 9310 and 9313. In the end, one of the colonies was stocked.

IMK771 (Fig. 1) was constructed by transforming CBS1483 by electroporation with $200 \mathrm{ng}$ of pUDP012 and $1 \mu \mathrm{g}$ of $120 \mathrm{bp}$ repair product obtained by mixing an equimolar amount of primers 10686 and 10687. Transformants were selected on SM-Ac plates. Deletion of SeILV6 was confirmed using primers 9310 and 9313. Prior stocking the isolate was successively streaked out on SM-Ac, YPD and SM-FAc plates. Genotype was systematically verified after each plating round with primers 9310 and 9313. In the end, one of the colonies was stocked and sequenced.

IMK786 (Fig. 1) was constructed by transforming CBS1483 by electroporation with $200 \mathrm{ng}$ of pUDP044 and $1 \mu \mathrm{g}$ of $120 \mathrm{bp}$ repair product obtained by mixing an equimolar quantity of primers 10992 and 10993 for SeATF1 and $1 \mu \mathrm{g}$ of $120 \mathrm{bp}$ repair product obtained by mixing an equimolar quantity of primers 10994 and 10995 for SeATF2. Transformants were selected on SM-Ac plates, deletion of SeATF1 and SeATF2 was confirmed using primers $11000 / 11001$ and primers $11002 / 11003$, respectively. Prior stocking the isolate was successively streaked out on SM-Ac, YPD and SM-FAc plates. Genotype was systematically verified after each plating round with primers pairs $11000 / 11001$ and $11002 / 11003$ to confirm SeATTF1 and SeATF2 deletions. In the end, one of the colonies was stocked.

IMK813 (Fig. 1) was constructed by transforming Weihenstephan $34 / 70$ by electroporation with $200 \mathrm{ng}$ of pUDP044 and $1 \mu \mathrm{g}$ of $120 \mathrm{bp}$ repair product obtained by mixing an equimolar quantity of primers 10992 and 10993 for SeATF1 and $1 \mu \mathrm{g}$ of 120 bp repair product obtained by mixing an equimolar quantity of primers 10994 and 10995 for SeATF2. Transformants 
were selected on SM-Ac plates, deletion of SeATF1 and SeATF2 was confirmed using primers 11000/11001 and primers 11002/11003, respectively.

\section{Next generation sequencing}

IMX1187 and IMK771 were incubated in $500-\mathrm{mL}$ shakeflasks containing $100 \mathrm{~mL}$ liquid YPD medium at $20{ }^{\circ} \mathrm{C}$ on an orbital shaker set at $200 \mathrm{rpm}$ until the strains reached stationary phase with an $\mathrm{OD}_{660}$ between 12 and 20. Genomic DNA for whole genome sequencing was isolated using the Qiagen 100/G kit (Qiagen, Hilden, Germany) according to the manufacturer's instructions and quantified using a Qubit ${ }^{\circledR}$ Fluorometer 2.0 (ThermoFisher Scientific). $51.57 \mu \mathrm{g}$ of genomic DNA from IMX1187 and $14.20 \mu \mathrm{g}$ from IMK771 was sequenced by Novogene Bioinformatics Technology Co., Ltd (Yuen Long, Hong Kong) on a HiSeq 2500 (Illumina, San Diego, CA) with 150 bp paired-end reads using True-seq PCRfree library preparation (Illumina). CRISPR-Cas9 assisted deletions were verified by mapping the sequencing reads onto the S. pastorianus CBS1483 genome [4] using the Burrows-Wheeler Alignment tool (BWA) and further processed using SAMtools [41, 42]. The deletions were confirmed by visualising the generated bam files in the Integrative Genomics Viewer (IGV) software [43]. The sequencing data are available at NCBI (https://www.ncbi. nlm.nih.gov/) under the Bioproject PRJNA397648.

\section{Results}

\section{Construction of a S. pastorianus strain expressing cas 9}

To limit construct instability and facilitate successive genome editing events, a copy of the Streptococcus pyo-

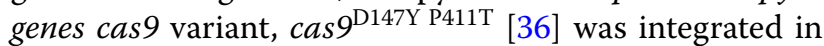
the genome of S. pastorianus CBS1483. The S. cerevisiae SPR3/YGR059W gene is involved in sporulation: a function impaired in S. pastorianus; therefore it was chosen as integration site as the impact on growth of deletion of SPR3 should be negligible. Additionally, SPR3 is located in the middle of the right arm of the S. cerevisiae CHRVII which counts only one copy in CBS1483, which should enable stable integration of a single cas 9 copy [4]. To prevent off-target integration driven by homology of the promoter and terminator, cas 9 was placed under the control of the TEF1 promoter from Arxula adeninivorans, which had been shown to be functional in Saccharomyces yeast [44]. The nourseothricin acetyl transferase expression cassette natNT2 expressed from the TEF1 promoter from the yeast Ashbya gossypii was used as a marker to select for integration [45] (Fig. 3). To guide the chromosomal integration of the endonuclease construct, the cas 9 containing fragment was flanked with an homology region of 480-bp targeting the SPR3 promoter region (HRL, Fig. 3a) and a 506-bp targeting the SPR3 terminator region (HRM) to complete the double cross over integration (Fig. 3a). These elements were assembled into a transformation cassette on pUD526 and the purified integration fragment was used to transform $S$. pastorianus CBS1483 yielding 14 transformants. In comparison, the same transformation in the laboratory S. cerevisiae CEN.PK113-7D yielded 476 transformants. Both transformations were performed simultaneously and under identical experimental conditions, therefore the difference in obtained transformants reflected the strong resilience of industrial S. pastorianus strains to transformation. The presence of the integrated construct was confirmed in all four tested colonies by PCR using specific primers (3750 and 9394) which amplify between the left homology arm for SPR3 and the end of the AaTEF1 promoter. Unexpectedly, a PCR targeting the SPR3 open reading frame using primers 3750 and 10435 yielded a fragment size corresponding to the wild type. Concomitantly, PCRs targeting cas 9 confirmed the integration in CHRVII in all four tested transformants, suggesting that either SPR3 might have been duplicated prior to replacement of one of the copies by cas 9 , or the cassette was not integrated as intended. To resolve the recombined SPR3 locus map, one of the transformants, was renamed IMX1187 and resequenced using Illumina technology. Mapping of the IMX1187 Illumina pair reads $(2 \times 150 \mathrm{bp})$ on the CBS1483 reference genome sequence confirmed the presence of the S. cerevisiae SPR3 wild type locus, but it also revealed that the region used for the integration HRL and HRM, exhibited a sequence depth coverage twofold higher than the SPR3 open reading frame and the surrounding chromosomal region (Fig. 3c). In the meantime, mapping of the IMX1187 reads on the sequence of the deletion cassette including the cas 9 and nat genes confirmed the single integration of the transformed fragment. Additionally, absence of reads mapping the $\beta$-lactamase gene bla present on pUD526 excluded the possibility that the plasmid got mistakenly integrated in the genome. To demonstrate anchoring of the cassette into CHRVII, the reads that mapped to the SPR3 region and to the integration fragment containing cas 9 and nat (including corresponding paired reads) were extracted and assembled using SPAdes [46]. The assembly confirmed that the cassette was anchored in CHRVII and the obtained graph suggested that the cas $9 /$ nat cassette integrated by single crossover resulting in a duplication of the integration site HMR or HML and integration of the cas 9 cassette (Fig. 3). However, the integration cassette was fully integrated and should result in expression of Cas9.

In literature, there are conflicting reports about the physiological consequences of Cas9 expression in Saccharomyces cerevisiae, depending on the mode and tuning of 


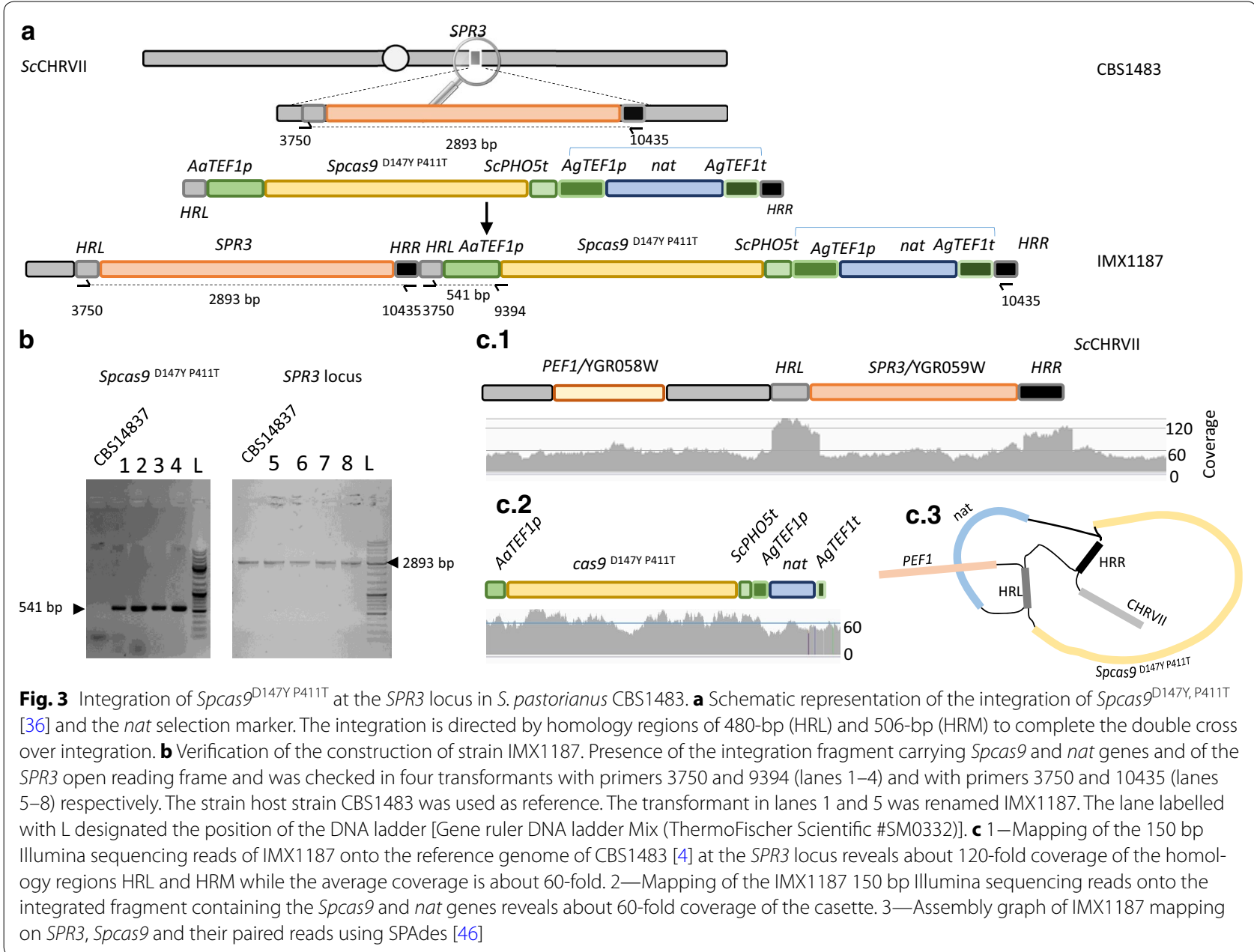

expression of the endonuclease gene $[15,19,47]$. Therefore, the growth rates of the S. pastorianus CBS1483 and IMX1187 (AaTEF1-cas9) were measured in YPD at $20^{\circ} \mathrm{C}$. The average maximum specific growth rate derived for biological triplicates for both strains did not deviate more than 2\%. The strains CBS1483 and IMX1187 exhibited growth rate of $0.263 \pm 0.002$ and $0.258 \pm 0.001 \mathrm{~h}^{-1}$ respectively (Fig. 4). This result confirmed that single integration of cas9 in CBS1483 (IMX1187) did not significantly affect the maximum specific growth rate.

\section{gRNA delivery systems for efficient editing in S. pastorianus}

After establishing the chromosomal integration of cas 9 in the genome of CBS1483, the next step consisted in demonstrating the activity of the RNA-programmed endonuclease. To do so, two gRNA delivery systems were tested, one based on the existing RNA polymerase III dependent system developed for S. cerevisiae [15] and one expression system based of ribozyme flanked gRNA expressed from a RNA polymerase II promoter. Firstly, the deletion

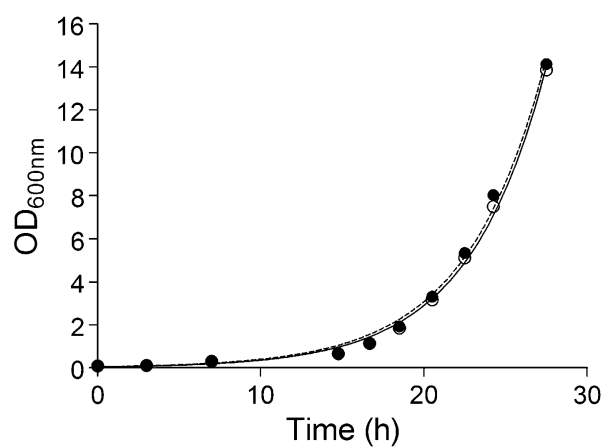

Fig. 4 Growth curve of the S. pastorianus strains CBS1483 (filled circle) and IMX1187 (Spcas9) (open circle). The strains CBS1483 and IMX1187 were strains were grown in complex medium (YPD) at $20^{\circ} \mathrm{C}$. Growth was monitored based on optical density at $660 \mathrm{~nm}\left(\mathrm{OD}_{660}\right)$ measurements. The data plotted are average and standard deviation of three biological replicates

of $U R A 3$ using the traditional RNA polymerase III system was tested in S. pastorianus strain IMX1187 (AaTEF1pcas 9 ). The selected 20-bp spacer to target URA3 matched 
the ScURA3 allele sequence perfectly (TTGACTGATTTTTCCATGGA), but carried one mismatch on the 12th position from its $3^{\prime}$ end (TTGACTGACTTTTCCATGGA) compared to the $S$. eubayanus allele (SeURA3). Both alleles shared the same gRNA spacer adjacent motif (PAM) sequence (GGG) and CBS1483 harbored three $S$. cerevisiae and two $S$. eubayanus alleles. The gRNA URA3 $_{3}$ was expressed by the RNA polymerase III dependent promoter $S N R 52 p[16,48]$ from the pROS12 plasmid, which carries a hygromycin resistance marker $h p h$ [15]. The resulting plasmid pUDR107 $\left(\mathrm{gRNA}_{\text {URA3 }}\right)$ was transformed in IMX1187 alone or together with two 120 bp double stranded repair DNA fragments for
ScURA3 and SeURA3. In absence of repair DNA, the transformation of the URA3 gRNA should in theory be lethal and yield few to no transformants, due to the inefficiency of non-homologous end joining (NHEJ) (Fig. 5a). However, the transformation of IMX1187 with pUDR107 alone returned several hundred of colonies, a number comparable to when the repair DNA was also provided. A set of ten clones from each transformation were picked and their genotype was diagnosed by specific PCR (9314 and 9317 for ScURA3 and 9318 and 9321 for SeURA3). All transformants either derived from the transformation with or without supply of a repair DNA produced a band with a size compatible with the wild type allele (Fig. 5b).

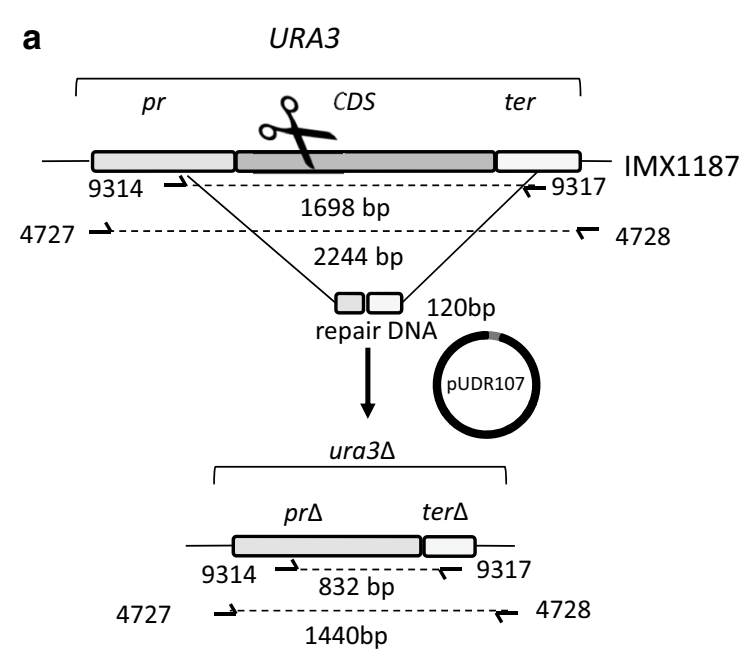

C

b

CBS1483SCURA3 Transf\# 1 Transf\#2 Transf\#3 Transf\# 4 Transf\# 5 Transf\# 6 Transf\# 7 Transf\# 8 Transf\# 9 Transf\#10

\section{gRNA spacer PAM}

TGGATATCTTGACTGATTTTTCCATGGAGGGCAC TGGATATCTTGACTGATTTTTCCATGGAGGGCAC TGGATATCTTGACTGATTTTTCCATGGA $\overline{G G G C A C}$ TGGATATCTTGACTGATTTTTCCATGGAGGGCAC TGGATATCTTGACTGATTTTTCCATGGA $\overline{G G G C A C}$ TGGATATCTTGACTGATTTTTCCATGGAGGGCAC TGGATATCTTGACTGATTTTTCCATGGA $\overline{G G G C A C}$ TGGATATCTTGACTGATTTTTCCATGGA GGGCAC TGGATATCTTGACTGATTTTTCCATGGA $\overline{G G G C A C}$ TGGATATCTTGACTGATTTTTCCATGGA $\overline{G G G C A C}$

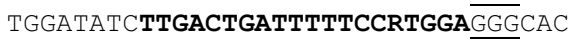
The

d
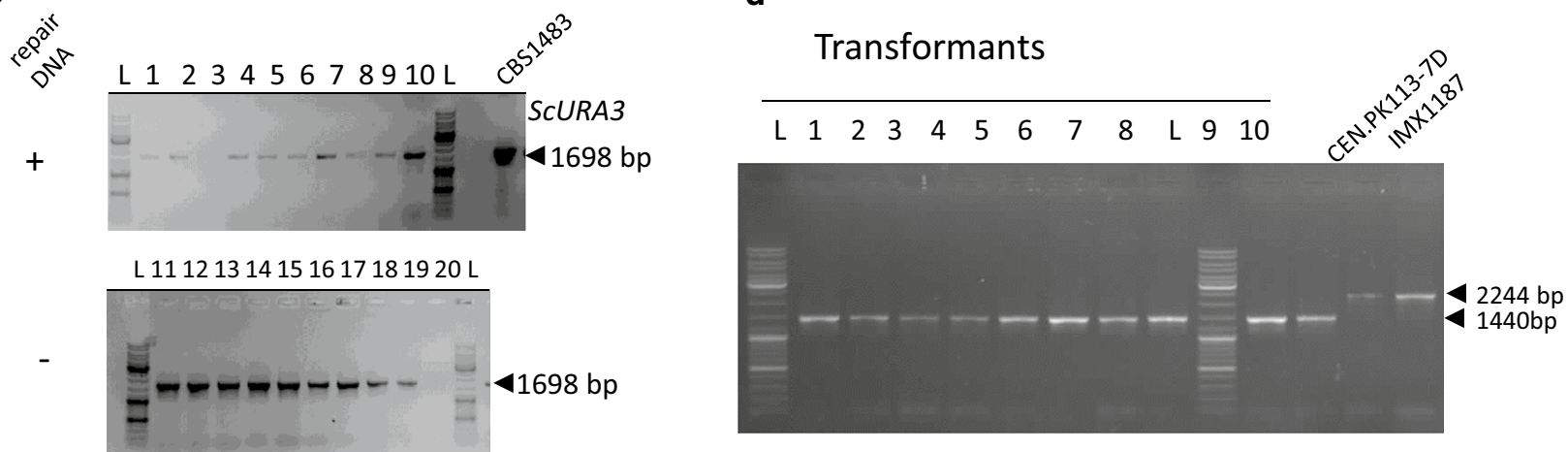

Fig. 5 Deletion of SCURA3 in IMX1187 and IMX585 using RNA III polymerase dependent (SNR52p) gRNA expression. a Representation of the native and deleted SCURA3. The plasmid pUDR107 carried a gRNA under the control of the SNR52p. Primers used for validation of the deletion are indicated. $\mathbf{b}$ Validation of transformants of the S. pastorianus IMX1187 strain with pUDR107 in presence or not of a 120 bp repair DNA. The PCR reactions were performed with the primers 9314 and 4728. All lanes (1-20) showed a PCR product of 1698 bp corresponding to the wildtype allele. The lane labelled with $L$ designated the position of the DNA ladder [Gene ruler DNA ladder Mix (ThermoFischer Scientific \#SM0332)]. c Sanger sequencing results of purified PCR fragments of ten transformants derived from the transformation of IMX1187 with pUD107 (gRNA $\left.A_{U R A}\right)$. The gRNA spacer used to direct Cas 9 is indicated in bold and the PAM sequence is underlined. $\mathbf{d}$ Validation of transformants of the S. cerevisiae IMX585 strain with pUDR107 in presence or not of a $120 \mathrm{bp}$ repair DNA. The PCR reactions were performed with the primers 4727 and 4728 . The lanes (1-10) corresponding to transformants obtained with repair DNA showed a PCR product of $1440 \mathrm{bp}$ corresponding to the deleted allele. The control lane labelled CEN. PK113-7D showed the wild type fragment at 2244 bp. The lane labelled with $L$ designated the position of the DNA ladder 
The Sanger sequencing results of the amplified fragments showed no indels at the site of the anticipated cut. With the exception of clone \#10 that showed an unresolved purine (R), all URA3 sequences were identical to that of the reference IMX1187, confirming the absence of editing (Fig. 5c). Therefore, to exclude defective expression of the gRNA, pUDR107 $\left(\mathrm{gRNA}_{U R A 3}\right)$ was also transformed in $S$. cerevisiae IMX585 (cas9) [15] together with the ScURA3 120 bp repair DNA. Out of the couple of dozens transformants, ten were randomly picked and diagnosed with by PCR. All transformants exhibited a band at $1440 \mathrm{bp}$ characteristic of the URA3 deletion. The same PCR from the untransformed CEN.PK113-7D yielded a fragment of $2244 \mathrm{bp}$ (Fig. 5d). This result established that pUDR107 enabled functional Cas9-mediated gene editing in S. cerevisiae IMX585, but not in S. pastorianus IMX1187.

While gRNA transcript level was not measured, RNA polymerase III expression is known to be low [49], a level which might be insufficient to enable efficient Cas9mediated introduction of a DSB. To circumvent this and to ensure high expression of the Cas9 programming RNA. In this approach, the gRNA was placed behind the control of the constitutive ScTDH3 promoter. To prevent modifications inherent to RNA polymerase II transcribed RNA, the gRNA was flanked by a Hammerhead ribozyme $(\mathrm{HH})$ and a Hepatitis Delta Virus ribozyme (HDV) on its $5^{\prime}$ and $3^{\prime}$ end respectively [21] (Fig. 6a). After transcription and self-cleavage of both ribozymes, high transcript levels of mature gRNA should be possible. Such an expression system was constructed, resulting in plasmid pUDP003, which harbored the $S$. cerevisiae codon optimized Aspergillus nidulans acetamidase gene (amdS) [34] and enabled insertion of a specific gRNA. This strategy was tested by attempting deletion of the SeILV6 gene in IMX1187 (AaTEF1p-cas9). The S. pastorianus strain CBS1483 and IMX1187 harbored only one ILV6 gene that originates from the $S$. eubayanus sub-genome [4]. The SeILV6 gene is located on the SeCHRIII, a chromosome present in four copies [4]. The gRNA SelLV6 $_{6}$ was inserted in plasmid pUDP003 (Fig. 2), resulting in plasmid pUDP010 (HH-gRNA SelLV6 -HDV amdS). Despite the absence of a S. cerevisiae ILV6 allele in IMX1187, the gRNA SeILV6 $_{\text {was }}$ designed to target ILV6 in S. cerevisiae as well. Thus, prior testing pUDP010 in S. pastorianus, the plasmid was transformed in S. cerevisiae IMX585. In the absence of a repair fragment, only 10 transformants were obtained while more than 500 were obtained when the repair fragment was included. Eventually a diagnostic PCR using specific primers confirmed successful deletion of ILV6 in IMX585 for all tested colonies. Similarly, transformation of pUDP010 (HH-gRNA ${ }_{\text {SelLV6 }}$-HDV amdS) in S. pastorianus IMX1187 (AaTEF1p-cas9) yielded 18 transformants when a $120 \mathrm{bp}$ repair fragment was co-transformed against just one when the repair fragment was omitted. Diagnostic PCR using primers 9310 and 9313 confirmed successful deletion of SeILV6 in IMX1187 for all tested colonies (Fig. 6c). It should be noted that the absence of bands of original size confirmed that all four copies of SeILV6 were deleted. The PCR characterization of the unique transformant obtained in absence of repair DNA indicated that the ILV6 locus was not deleted, since a band with a size compatible with the reference length was amplified, suggesting that the CRISPR-Cas9 induced DSB was repaired by NHEJ (Fig. 6c).

The ability to obtain successful deletion of ILV6 using the pUDP expression system indicated effective expression of the integrated cas 9 in S. pastorianus IMX1187, despite its imperfect integration in the SPR3 locus. The failure to obtain deletion of $U R A 3$ using the RNA polymerase III dependent gRNA expression system in $S$. pastorianus IMX1187 while deletion was possible in $S$. cerevisiae IMX585 indicated that this gRNA expression system was not effective in S. pastorianus. Based on literature, this ineffectiveness may be caused by low gRNA transcripts levels. Regardless, the new pUDP expression system was functional in $S$. pastorianus and the deletion of ILV6 constituted the first reported successful use of Cas9 engineering in S. pastorianus.

\section{Plasmid-based co-expression of Cas9 and gRNA in S. \\ pastorianus}

Given the notoriously low efficiency of gene insertion by homologous recombination in the genome of $S$. pastorianus, a plasmid was designed for co-expression of cas 9 together with the gRNA, which would render cas 9 expression more reproducible and facilitate genome editing in different S. pastorianus strains. The plasmid pUDP004 combined the cas 9 expression cassette previously integrated in IMX1187 and the different elements of pUDP003 including the RNA polymerase II dependent gRNA expression cassette (Fig. 2). To assess the efficacy of the pUDP004 system relative to the chromosome borne cas 9 together with the pUDP003 system, $\mathrm{gRNA}_{\text {SelLV6 }}$ was inserted in pUDP004 and the resulting plasmid pUDP012 was used to transform CBS1483. In absence of a 120-bp repair DNA, a total of 14 transformants were obtained, while the number of transformants increased by 63 -fold reaching a total of 884 transformants when the repair fragment was co-transformed. Diagnostic PCR using primers 9310 and 9313 confirmed successful deletion of SeILV6 in for all tested colonies and one colony producing a fragment corresponding to effective deletion of SeILV6 was stocked as IMK771. To eliminate any doubt, the IMK771 genome was resequenced using Illumina sequencing technology. The 150 -bp pair-end 


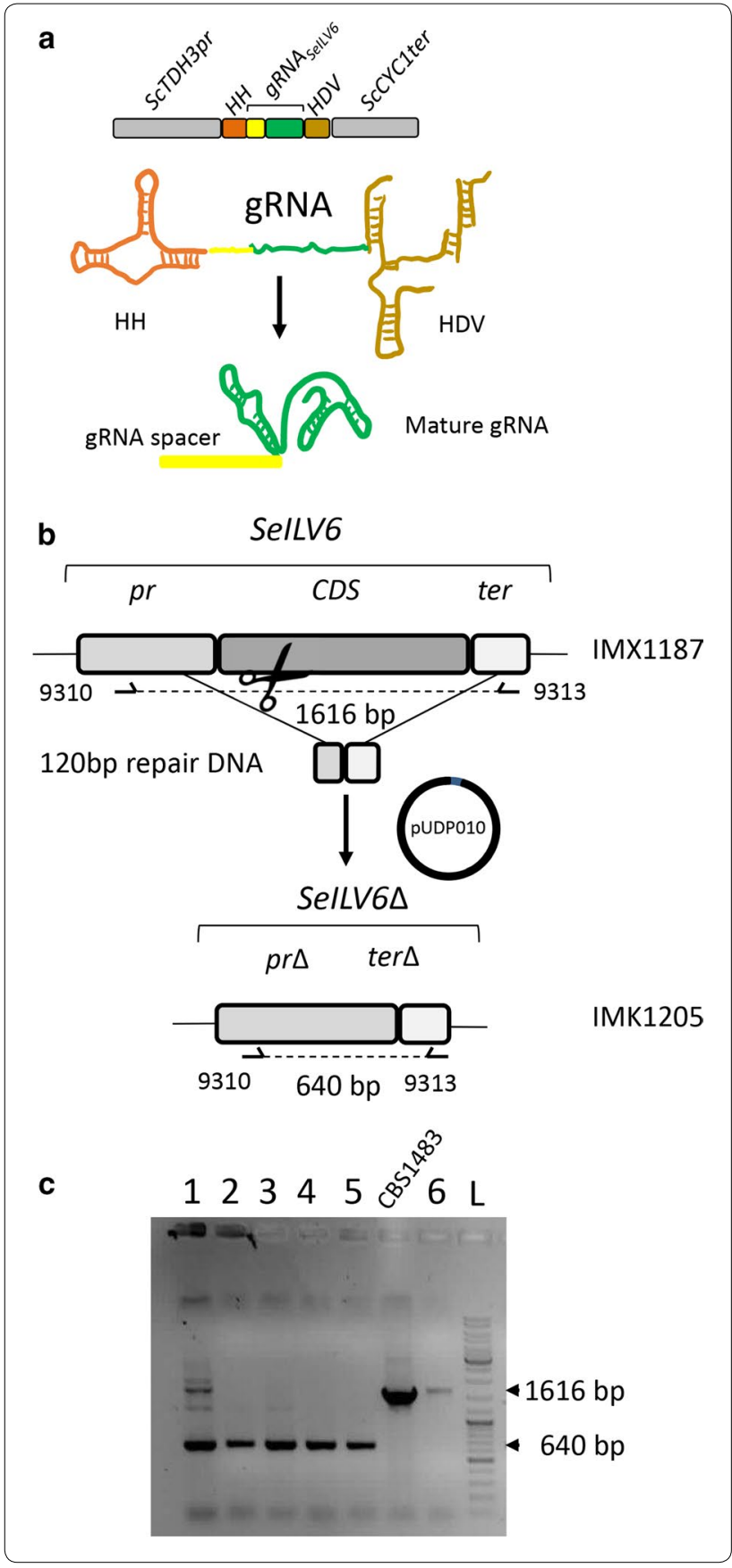

reads were mapped on the CBS1483 reference genome sequence [4] and as expected no reads mapped to the region targeted for deletion, indicating complete deletion of all four alleles of SeILV6. These results demonstrated that the plasmid-based co-expression of cas 9 and a gRNA was functional and could be used for effective genome editing in S. pastorianus.
Fig. 6 Ribozymes flanked gRNA driven deletion of SellV6 in S. pastorianus IMX1187. a Representation of the gRNA expression cassette in pUDP010. The gRNA sellv6 $_{\text {was }}$ wlanked on its $5^{\prime}$ by a hammerhead ribozyme ( $\mathrm{HH}$ represented in orange) and on its $3^{\prime}$ by a hepatitis delta virus (HDV represented in bronze) ribozyme. This construct was under the control of the RNA polymerase II promoter SCTDH3 and the $\mathrm{ScCYC1}$ terminator. Upon ribozyme self-cleavage, a mature gRNA comprising the SellV6 guiding spacer (in yellow) and the constant structural gRNA fragment (in green) is released. b Schematic representation of the SelLV6 editing upon transformation of IMX1187 with pUDP010. The primers for the validation of transformants are indicated. c Validation of transformants of the S. pastorianus IMX1187 strain with pUDP010 in presence of a $120 \mathrm{bp}$ repair DNA. The lanes $(1-5)$ corresponding to the transformants obtained with repair DNA showed a PCR product of $640 \mathrm{bp}$ corresponding to the deleted allele. One of the transformants exhibiting an SellVV deletion was renamed IMK1205. The control lane labelled CBS1483 and lane 6 corresponding to one transformant obtained without repair DNA showed a PCR product corresponding to the wild type fragment at $1616 \mathrm{bp}$. The lane labelled with $L$ designated the position of the DNA ladder [Gene ruler DNA ladder Mix (ThermoFischer Scientific \#SM0332)]

\section{Multiplexing gene targeting by expression of double ribozyme flanked $g$ RNAs array}

Despite the preexisting good genetic accessibility of $S$. cerevisiae strains, CRISPR-Cas 9 mediated editing greatly simplified genome engineering approaches. In particular, the ability to multiplex editing events $[15,18,50]$. Therefore, the possibility of multiplexed gRNA expression was investigated in the pUDP expression system. Conveniently, the self-cleaving properties of the ribozymes might be compatible with the construction of adjacent HH-gRNA-HDV linked in a polycistronic array.

Encouraged by the successful SeILV6 deletion using pUDP004 based gRNA expression, a tandem array of [HH-gRNA-HDV] targeting SeATF1 and SeATF2 in S. pastorianus was designed. The two $\mathrm{HH}$-gRNA-HDV were spaced with a 10-bp linker. The synthesized array was placed under the control of the ScTDH3 promoter in pUDP004 as described earlier for the SeILV6 gene. The recombinant plasmid pUDP044 (amdS cas9 TDH3p$\mathrm{HH}$-gRNA ${ }_{S e A T F 1}-\mathrm{HDV}-\mathrm{HH}$-gRNA $\left.{ }_{S e A T F 2}-\mathrm{HDV}-C Y C 1 \mathrm{t}\right)$ was then used to transform two $S$. pastorianus strains: CBS1483 and Weihenstephan 34/70 (Fig. 7a). CBS1483 harboured one and three copies of SeATF1 and SeATF2 respectively, while Weihenstephan $34 / 70$ missed one SeATF2 allele relative to CBS1483. Co-transformation of CBS1483 and Weihenstephan 34/70 with pUPD044 and the corresponding repair fragments yielded 43 and 189 transformants per plate respectively. In the absence of repair fragments, 15 and 44 colonies were obtained in CBS1483 and Weihenstephan 34/70, respectively. A 


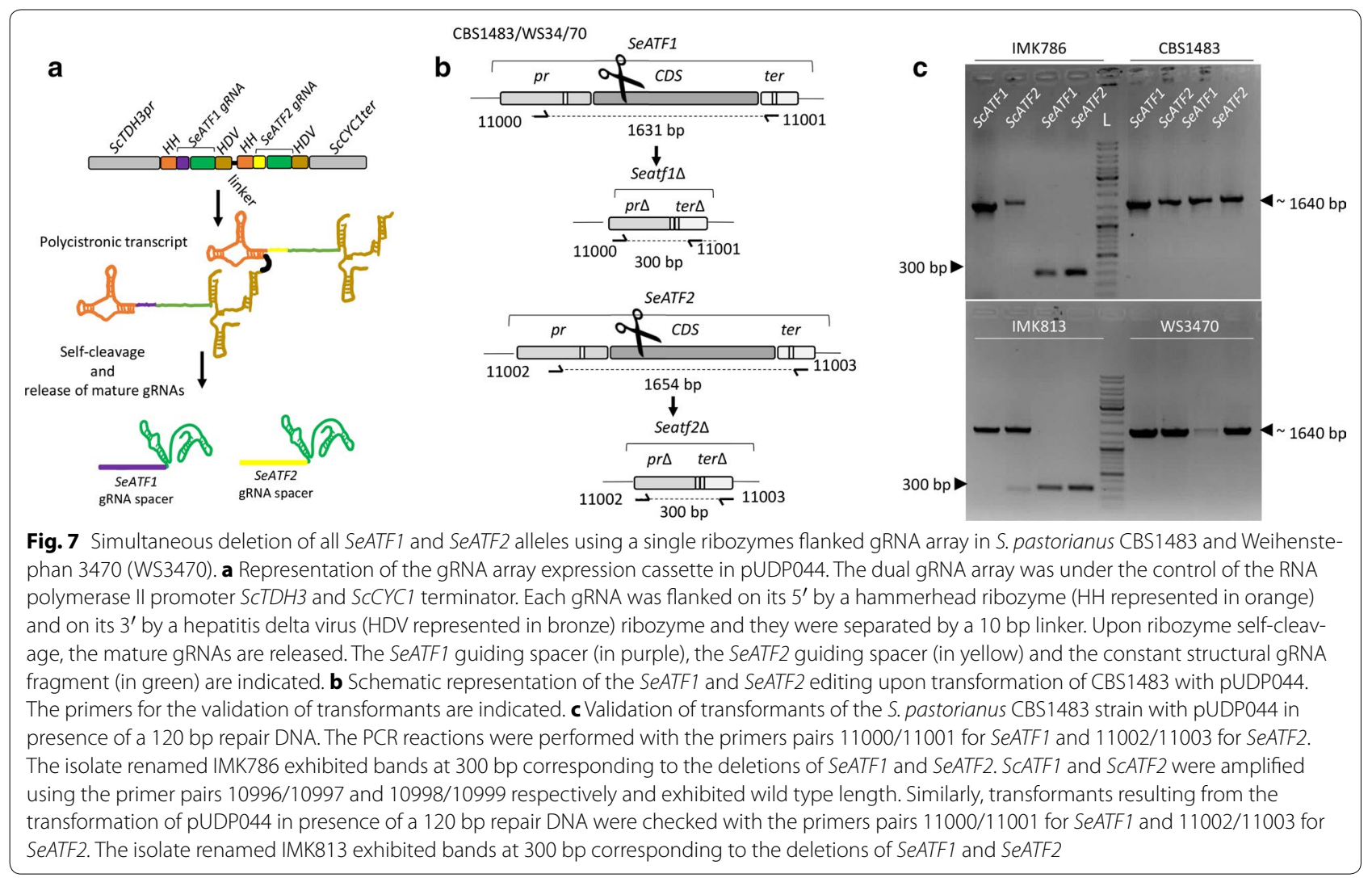

randomly picked set of seven colonies transformed with repair fragment were verified by PCR, which confirmed that all copies of SeATF1 and SeATF2 were deleted. One of the CBS1483 transformants exhibiting the correct double SeATF1/SeAFT2 deletion was named IMK786 and similarly a Weihenstephan transformant was named IMK813 (Fig. 7). The designed gRNAs were also confirmed to be specific to the $S$. eubayanus genes as the ScATF1 and ScATF2 genes were not affected (Fig. 7c). To the best of our knowledge, this represents the first application of polycistronic ribozyme flanked gRNA, as well as the first demonstration of a successful double deletion in S. pastorianus.

\section{Discussion}

\section{Saccharomyces pastorianus is not genetically amendable}

The results reported in this study firmly established that CRISPR-Cas9 improves the performance of homologydirected recombination in $S$. pastorianus. In contrast to $S$. cerevisiae, a species amenable to genetic modification, the interspecific hybrid S. pastorianus has shown higher resilience to targeted genetic alterations. This was exemplified by the attempt to integrate the cas 9 gene at a specific chromosomal site using traditional double cross over. The size of the cassette complicated the genotype characterisation, but the presence of the endonuclease gene was confirmed and although whole genome resequencing of the strain IMX1187 did not completely resolve the structure of the recombined locus, it strongly suggested that a single crossover integration event occurred, resulting in integration of cas 9 next to SPR3 instead of replacing SPR3 as intended. Several literature reports corroborated our unfortunate experience $[7,8]$. In different microbial systems, the efficiency of integration by homologous recombination was improved by impairing the non-homologous end-joining (NHEJ) function [51-53]. This approach, though successful, was often accompanied by side effects such as an exacerbated sensitivity to environmental stresses. In S. cerevisiae, inactivation of Yku70 and Yku80, two proteins involved in NHEJ, resulted in severe alterations of telomere maintenance and function as well as in deregulation of the cell cycle [54-58], which might explain why this strategy has never been attempted in S. pastorianus. Furthermore, the absence of improvement of the S. pastorianus genetic accessibility is not so surprising after all, since the brewing industry as most industries involved in fermentation of products intended for human consumption, has been reluctant to apply genetically modified organisms by fear of consumers group opinion [59], and has privileged classical strain improvement programmes. 
Eventually, the results reported in this study demonstrated that the introduction of a DSB, which stimulates occurrence of homologous recombination, would represent an efficient solution to circumvent the natural resilience to targeted genetic modification in S. pastorianus.

\section{gRNA expression in S. pastorianus}

Editing systems developed for $S$. cerevisiae could not be directly transfered to $S$. pastorianus. Although convoluted, the functionality of Cas9 in S. pastorianus was eventually demonstrated. In contrast to the situation in S. cerevisiae, the expression of the gRNA from the SNR52 promoter was unsuccessful. While the objectives of the study were not to fully understand the origin of the lack of functionality of the SNR52 driven gRNA expression, we could hypothesize that this problem might arise from the hybrid genome composition of $S$. pastorianus. Their alloaneuploid genome is a source of genetic innovations, e.g. increased chromosome copy number has facilitated introduction of allelic variations and cohabitation of the two parental genomes might have stimulated the adjustment of transcription circuits which together have contributed to adaptation of lager yeast to the intensified brewing environment [4, 60, 61]. Furthermore, many cellular functions are controlled by protein complexes which in hybrid strains may be formed by assemblies of subunits originating from both parental sub-genomes, thereby creating another source of variation [62]. The RNA polymerase III is a complex formed of six different subunits (TFC1, 3, 4, 6, 7 and 8) and the strain CBS1483 retained both parental gene sets [4]. Thus, the absence of editing might reflect a modification of the RNA polymerase III transcriptional control in S. pastorianus relative to S. cerevisiae. This could also be associated with promoter sequence variations between the parents and the hybrid. The inspection of the SNR52 promoter sequences of the S. cerevisiae and S. eubayanus parents revealed nucleotide variations with S. pastorianus promoters (Fig. 8). The ScSNR52 promoter from CBS1483 carried one mutation in position - 4 (G to A), while the CBS1483 SeSNR52 promoter exhibited four single nucleotide variations with two located between the positions -1 and -100 . In all configurations, the absence of editing points towards too low gRNA expression.

Fortunately, the proposed alternative involving expression of a ribozyme protected gRNA system turned out to be successful. In this method already used in human cells [63], plants [21, 32] and fungi $[64,65]$ the gRNA construct is expressed from a RNA polymerase II promoter. All CRISPR-Cas9 assisted deletions attempted (SeILV6,

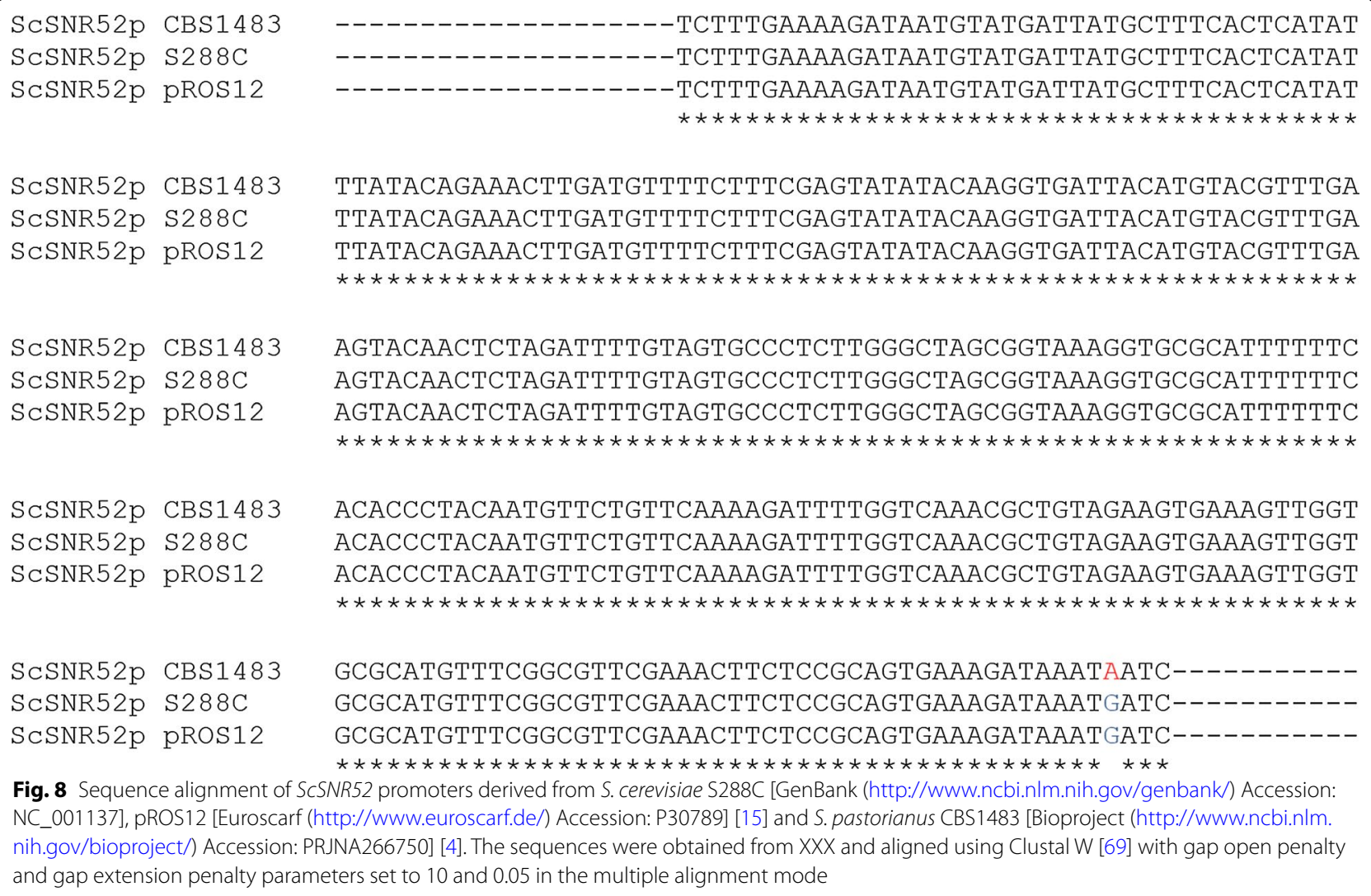

Fig. 8 Sequence alignment of SCSNR52 promoters derived from S. cerevisiae S288C [GenBank (http://www.ncbi.nlm.nih.gov/genbank/) Accession: NC_001137], pROS12 [Euroscarf (http://www.euroscarf.de/) Accession: P30789] [15] and S. pastorianus CBS1483 [Bioproject (http://www.ncbi.nlm. nih.gov/bioproject/) Accession: PRJNA266750] [4]. The sequences were obtained from XXX and aligned using Clustal W [69] with gap open penalty and gap extension penalty parameters set to 10 and 0.05 in the multiple alignment mode 
SeATF1 and SeATF2) were introduced with high fidelity. The challenging nature of genetic modification in $S$. pastorianus does not come only from the low efficiency of homologous recombination, but also from the requirement to delete multiple alleles simultaneously due to its extensive aneuploidy [61]. In the case of SeILV6, four alleles were simultaneously deleted without introduction of any markers at the loci. The fact that all alleles were deleted at once as intended demonstrates the potency of a CRISPR-Cas9 induced DSB to stimulate targeted homology-mediated integration and circumvent unreliability of recombination in S. pastorianus. Remarkably, this could be achieved simultaneously at two different chromosomal loci (SeATF1 on CHR SeVIII-SeXV and SeATF2 CHR SeVII-ScVII) as well [4]. In total, this resulted in the deletion of five different alleles, one short to the highest number of simultaneously completed deletions in S. cerevisiae [15]. While previously suggested [21, 63], polycistronic ribozymes flanked gRNA expressed from a RNA pol II promoter had never been assayed before. Our results experimentally confirmed that $5^{\prime}$ and $3^{\prime}$ extension as designed at the junction of the two gRNA cassettes did not hinder self-cleavage of $\mathrm{HH}$ and HDV ribozymes and allow release of functional mature gRNAs. This result provided a glimpse of the potential of this mode of expression. It would suggest that construction of polycistronic array including more than two gRNA could be contemplated.

\section{Expanding the S. pastorianus genetic tool box}

The present study delivered the first really efficient technical solution readily useable to perform targeted genetic modifications in S. pastorianus. The functionality of two modes of Cas9 expression was shown. Chromosomal integration of cas9 (IMX1187) coupled with plasmidbased gRNA expression might be privileged when successive transformations are foreseen [7]. However, plasmid-based cas 9 and gRNA co-expression proved to be as effective and presents the advantage to be easily transferable in multiple strain backgrounds. For efficient use of the provided repair fragment to recombine at the locus of the Cas9-induced DSB, Cas9 activity and presence of the repair DNA have to be synchronous. The correct integration of the repair fragment during single and double gene editing showed that the endonuclease was transcribed and translated fast enough for free linear DNA to still be available for repair of the induced DSB. These outcomes were in line with similar approaches attempted in S. cerevisiae or in Aspergillus niger [36, 47, 64]. The presence of the gRNA is not constantly needed, as soon as the chromosomal double cut is inserted and preferably repaired, the plasmid has to be lost to recover a plasmid-free modified strain to either test the strain physiology or to prepare the constructed strain for a next editing round. The selection marker and replication origin used in the pUDP expression system tested in this work were designed to be broadly applicable and to facilitate rapid plasmid recycling. The dominant acetamidase marker confers the ability to use acetamide as sole nitrogen source and can be used in prototrophic strains such as lager yeasts or more generally industrial $\mathrm{Sac}$ charomyces strains. Plasmids carrying the amdS marker can be counter selected by growth in presence of fluoroacetamide [34]. Additionally the panARSopt replication origin [40]) derived from $K$. lactis used in the pUDP expression system was shown to be functional in a wide range of yeast species including S. cerevisiae. Contrarily to most replication origins such as the $2 \mu \mathrm{m}$ replication origin, which necessitates the presence of a wild type native $2 \mu \mathrm{m}$ plasmid to provide the enzymatic replicative machinery, panARSopt does not require any other genetic element. Furthermore, like ARS-CEN-based plasmids, panARS-based plasmids showed loss frequencies ranging between 5 and $10 \%$ per generation when grown in non-selective conditions [40]. These properties should permit efficient use of the pUDP expression system in various strain backgrounds, which might help to standardize a genome editing protocol starting from the design and cloning of the gRNA to the selection of correctly edited strains which have lost the pUDP plasmid.

Finally, while the scope of this work limited the tools application to single and double gene deletions, the availability of CRISPR-Cas9 editing tool makes a broad range of genetic modifications possible. Analogously to modification techniques applied in S. cerevisiae, the pUDP expression system might be applied for in vivo site directed mutagenesis and targeted introduction of multiple genes or entirely new pathways. In S. pastorianus, such modifications would finally allow to systematically investigate the contribution of genes involved in brewing-relevant phenotypes of S. pastorianus. In particular, the use of subgenome specific gRNA targets could enable targeted modification of genes from the S. cerevisiae and S. eubayanus subgenomes and thereby enable research on their interaction. For example, elucidation of the role of individual flocculation genes or implication of individual maltose and maltotriose transporter in S. pastorianus could now be envisaged.

\section{Conclusions}

The gRNA and Cas9 expression system developed in this study enabled CRISPR-Cas9 engineering in S. pastorianus. The system was applied successfully for the deletion of all alleles of SeILV6 and could be multiplexed successfully to obtain the simultaneous deletion of all alleles of SeATF1 and SeATF2. While the system was 
only tested for gene deletion in this study, functional CRISPR-Cas9 engineering in S. pastorianus should also facilitate approaches such as gene insertions and directed mutagenesis. As $S$. pastorianus is notoriously resilient to genetic modification, these developments significantly improve its genetic accessibility and facilitate future research into the complex allo-aneuploid genome of $S$. pastorianus.

\section{Authors' contributions}

PAdG performed plasmid and strain construction. ARGdV prepared genomic DNA for whole genome sequencing. ARGdV and MvdB performed bioinformatics analysis. JMGD designed the pUDP-based Cas9 modification system. ARGdV and JMGD supervised the study. ARGdV, PAdG and JMGD wrote the manuscript. All authors read and approved the final manuscript.

\section{Acknowledgements}

The authors would like to thank Hannes Jürgens (Delft University of Technology) and Veronica Gast (Delft University of Technology) for their contributions to the pUDP plasmid modification system. We also would like to thank Xavier Hakkaart for kindly providing pUDR107. We are thankful to Prof. JackT. Pronk (Delft University of Technology) and Dr. Niels Kuijpers (HEINEKEN Supply Chain B.V.) for their support during this project and their critical reading of the manuscript.

\section{Competing interests}

The authors declare that they have no competing interests.

\section{Availability of data and materials}

The raw sequencing data of IMX1187 and IMK771 are available at NCBI under the Bioproject PRJNA397648. Plasmids pUDP003, pUDP004. pUDP010, pUDP012 and pUDP044 were deposited at addgene (http://www.addgene. org/).

\section{Consent for publication \\ Not applicable.}

Ethics approval and consent to participate Not applicable.

\section{Funding}

This work was performed within the BE-Basic R\&D Program (http://www. be-basic.org/), which was granted an FES subsidy from the Dutch Ministry of Economic Affairs, Agriculture and Innovation (EL\&l).

\section{Publisher's Note}

Springer Nature remains neutral with regard to jurisdictional claims in published maps and institutional affiliations.

Received: 23 August 2017 Accepted: 23 November 2017

Published online: 05 December 2017

\section{References}

1. Libkind D, Hittinger CT, Valerio E, Goncalves C, Dover J, Johnston M, Goncalves P, Sampaio JP. Microbe domestication and the identification of the wild genetic stock of lager-brewing yeast. Proc Natl Acad Sci USA. 2011;108:14539-44

2. Nakao Y, Kanamori T, Itoh T, Kodama Y, Rainieri S, Nakamura N, Shimonaga T, Hattori M, Ashikari T. Genome sequence of the lager brewing yeast, an interspecies hybrid. DNA Res. 2009;16:115-29.

3. Okuno M, Kajitani R, Ryusui R, Morimoto H, Kodama Y, Itoh T. Next-generation sequencing analysis of lager brewing yeast strains reveals the evolutionary history of interspecies hybridization. DNA Res. 2016;23:67-80.
4. van den Broek M, Bolat I, Nijkamp JF, Ramos E, Luttik MAH, Koopman F, Geertman JM, de Ridder D, Pronk JT, Daran JM. Chromosomal copy number variation in Saccharomyces pastorianus is evidence for extensive genome dynamics in industrial lager brewing strains. Appl Environ Microbiol. 2015:81:6253-67.

5. Walther A, Hesselbart A, Wendland J. Genome sequence of Saccharomyces carlsbergensis, the world's first pure culture lager yeast. G3. 2014;4:783-93

6. Hewitt SK, Donaldson IJ, Lovell SC, Delneri D. Sequencing and charac terisation of rearrangements in three S. pastorianus strains reveals the presence of chimeric genes and gives evidence of breakpoint reuse. PLoS ONE. 2014;9:e92203.

7. Duong CT, Strack L, Futschik M, Katou Y, Nakao Y, Fujimura T, Shirahige K, Kodama Y, Nevoigt E. Identification of Sc-type ILV6 as a target to reduce diacetyl formation in lager brewers'yeast. Metab Eng. 2011;13:638-47.

8. Bolat I, Romagnoli G, Zhu F, Pronk JT, Daran JM. Functional analysis and transcriptional regulation of two orthologs of ARO10, encoding broadsubstrate-specificity 2-oxo-acid decarboxylases, in the brewing yeast Saccharomyces pastorianus CBS1483. FEMS Yeast Res. 2013;13:505-17.

9. Murakami N, Miyoshi S, Yokoyama R, Hoshida H, Akada R, Ogata T. Construction of a URA3 deletion strain from the allotetraploid bottomfermenting yeast Saccharomyces pastorianus. Yeast. 2012;29:155-65.

10. Bollag RJ, Waldman AS, Liskay RM. Homologous recombination in mammalian cells. Annu Rev Genet. 1989;23:199-225.

11. Choulika A, Perrin A, Dujon B, Nicolas JF. Induction of homologous recombination in mammalian chromosomes by using the I-Scel system of Saccharomyces cerevisiae. Mol Cell Biol. 1995;15:1968-73.

12. Kuijpers NGA, Solis-Escalante D, Bosman L, van den Broek M, Pronk JT, Daran JM, Daran-Lapujade P. A versatile, efficient strategy for assembly of multi-fragment expression vectors in Saccharomyces cerevisiae using 60 bp synthetic recombination sequences. Microb Cell Fact. 2013;12:47.

13. Jinek M, Chylinski K, Fonfara I, Hauer M, Doudna JA, Charpentier E. A programmable dual-RNA-guided DNA endonuclease in adaptive bacterial immunity. Science. 2012;337:816-21.

14. Cong L, Ran FA, Cox D, Lin S, Barretto R, Habib N, Hsu PD, Wu X, Jiang W, Marraffini LA, Zhang F. Multiplex genome engineering using CRISPR/Cas systems. Science. 2013;339:819-23.

15. Mans R, van Rossum HM, Wijsman M, Backx A, Kuijpers NGA, van den Broek M, Daran-Lapujade P, Pronk JT, van Maris AJA, Daran JMG. CRISPR/ Cas9: a molecular Swiss army knife for simultaneous introduction of multiple genetic modifications in Saccharomyces cerevisiae. FEMS Yeast Res. 2015;15:fov004

16. DiCarlo JE, Norville JE, Mali P, Rios X, Aach J, Church GM. Genome engineering in Saccharomyces cerevisiae using CRISPR-Cas systems. Nucleic Acids Res. 2013;41:4336-43.

17. Jakociunas T, Rajkumar AS, Zhang J, Arsovska D, Rodriguez A, Jendresen CB, Skjodt ML, Nielsen AT, Borodina I, Jensen MK, Keasling JD. CasEMBLR: Cas9-facilitated multiloci genomic integration of in vivo assembled DNA parts in Saccharomyces cerevisiae. ACS Synth Biol. 2015;4:1226-34.

18. Horwitz AA, Walter JM, Schubert MG, Kung SH, Hawkins K, Platt DM, Hernday AD, Mahatdejkul-Meadows T, Szeto W, Chandran SS, Newman JD. Efficient multiplexed integration of synergistic alleles and metabolic pathways in yeasts via CRISPR-Cas. Cell Syst. 2015;1:88-96.

19. Ryan OW, Cate JHD. Multiplex engineering of industrial yeast genomes using CRISPRm. Method Enzymol. 2014:546:473-89.

20. Turowski TW, Tollervey D. Transcription by RNA polymerase III: insights into mechanism and regulation. Biochem Soc Trans. 2016:44:1367-75.

21. Gao Y, Zhao Y. Self-processing of ribozyme-flanked RNAs into guide RNAs in vitro and in vivo for CRISPR-mediated genome editing. J Int Plant Biol. 2014;56:343-9.

22. Mali P, Yang L, Esvelt KM, Aach J, Guell M, DiCarlo JE, Norville JE, Church GM. RNA-guided human genome engineering via Cas9. Science. 2013;339:823-6.

23. Li W, Teng F, LiT, Zhou Q. Simultaneous generation and germline transmission of multiple gene mutations in rat using CRISPR-Cas systems. Nat Biotechnol. 2013:31:684-6.

24. Hwang WY, Fu Y, Reyon D, Maeder ML, Tsai SQ, Sander JD, Peterson RT, Yeh JR, Joung JK. Efficient genome editing in zebrafish using a CRISPRCas system. Nat Biotechnol. 2013;31:227-9. 
25. Friedland AE, Tzur YB, Esvelt KM, Colaiacovo MP, Church GM, Calarco JA. Heritable genome editing in C. elegans via a CRISPR-Cas9 system. Nat Methods. 2013;10:741-3.

26. Waaijers S, Portegijs V, Kerver J, Lemmens BB, Tijsterman M, van den Heuvel S, Boxem M. CRISPR/Cas9-targeted mutagenesis in Caenorhabditis elegans. Genetics. 2013;195:1187-91.

27. Gratz SJ, Cummings AM, Nguyen JN, Hamm DC, Donohue LK, Harrison MM, Wildonger J, O'Connor-Giles KM. Genome engineering of Drosophila with the CRISPR RNA-guided Cas9 nuclease. Genetics. 2013;194:1029-35.

28. Schwartz CM, Hussain MS, Blenner M, Wheeldon I. Synthetic RNA polymerase III promoters facilitate high-efficiency CRISPR-Cas9-mediated genome editing in Yarrowia lipolytica. ACS Synth Biol. 2016;5:356-9.

29. Weninger A, Hatzl AM, Schmid C, Vogl T, Glieder A. Combinatorial optimization of CRISPR/Cas9 expression enables precision genome engineering in the methylotrophic yeast Pichia pastoris. J Biotechnol. 2016;235:139-49.

30. Feng Z, Zhang B, Ding W, Liu X, Yang DL, Wei P, Cao F, Zhu S, Zhang F, Mao $Y$, Zhu JK. Efficient genome editing in plants using a CRISPR/Cas system. Cell Res. 2013;23:1229-32

31. Zhang B, Yang X, Yang CP, Li MY, Guo YL. Exploiting the CRISPR/Cas9 system for targeted genome mutagenesis in petunia. Sci Rep. 2016;6:20315.

32. Miao J, Guo D, Zhang J, Huang Q, Qin G, Zhang X, Wan J, Gu H, Qu LJ. Targeted mutagenesis in rice using CRISPR-Cas system. Cell Res. 2013;23:1233-6.

33. Verduyn C, Postma E, Scheffers WA, Vandijken JP. Effect of benzoic-acid on metabolic fluxes in yeasts - a continuous-culture study on the regulation of respiration and alcoholic fermentation. Yeast. 1992;8:501-17.

34. Solis-Escalante D, Kuijpers NG, Bongaerts N, Bolat I, Bosman L, Pronk JT, Daran JM, Daran-Lapujade P. amdSYM, a new dominant recyclable marker cassette for Saccharomyces cerevisiae. FEMS Yeast Res. 2013;13:126-39.

35. Thompson JR, Register E, Curotto J, Kurtz M, Kelly R. An improved protocol for the preparation of yeast cells for transformation by electroporation. Yeast. 1998;14:565-71.

36. Bao ZH, Xiao H, Lang J, Zhang L, Xiong X, Sun N, Si T, Zhao HM. Homology-integrated CRISPR-Cas (HI-CRISPR) system for one-step multigene disruption in Saccharomyces cerevisiae. ACS Synth Biol. 2015;4:585-94.

37. Yanisch-Perron C, Vieira J, Messing J. Improved M13 phage cloning vectors and host strains: nucleotide sequences of the M13mp18 and pUC19 vectors. Gene. 1985;33:103-19.

38. de Kok S, Stanton LH, Slaby T, Durot M, Holmes VF, Patel KG, Platt D, Shapland EB, Serber Z, Dean J, et al. Rapid and reliable DNA assembly via ligase cycling reaction. ACS Synth Biol. 2014;3:97-106.

39. Gibson DG, Young L, Chuang RY, Venter JC, Hutchison CA 3rd, Smith HO. Enzymatic assembly of DNA molecules up to several hundred kilobases. Nat Methods. 2009;6:343-5.

40. Liachko I, Dunham MJ. An autonomously replicating sequence for use in a wide range of budding yeasts. FEMS Yeast Res. 2014;14:364-7.

41. Li H, Durbin R. Fast and accurate long-read alignment with BurrowsWheeler transform. Bioinformatics. 2010;26:589-95.

42. Li H, Handsaker B, Wysoker A, Fennell T, Ruan J, Homer N, Marth G, Abecasis G, Durbin R, Genome Project Data Processing S. The sequence alignment/map format and SAMtools. Bioinformatics. 2009;25:2078-9.

43. Robinson JT, Thorvaldsdottir H, Winckler W, Guttman M, Lander ES, Getz G, Mesirov JP. Integrative genomics viewer. Nat Biotechnol. 2011;29:24-6.

44. Wartmann T, Boer E, Pico AH, Sieber H, Bartelsen O, Gellissen G, Kunze G. High-level production and secretion of recombinant proteins by Arxula adeninivorans. Yeast. 2003:20:S326.

45. Goldstein AL, McCusker JH. Three new dominant drug resistance cassettes for gene disruption in Saccharomyces cerevisiae. Yeast. 1999:15:1541-53.

46. Bankevich A, Nurk S, Antipov D, Gurevich AA, Dvorkin M, Kulikov AS, Lesin VM, Nikolenko SI, Pham S, Prjibelski AD, et al. SPAdes: a new genome assembly algorithm and its applications to single-cell sequencing. J Comput Biol. 2012;19:455-77.

47. Generoso WC, Gottardi M, Oreb M, Boles E. Simplified CRISPR-Cas genome editing for Saccharomyces cerevisiae. J Microbiol Methods. 2016;127:203-5.
48. Guffanti E, Ferrari R, Preti M, Forloni M, Harismendy O, Lefebvre O, Dieci G. A minimal promoter for TFIIIC-dependent in vitro transcription of snoRNA and tRNA genes by RNA polymerase III. J Biol Chem. 2006;281:23945-57.

49. Harismendy O, Gendrel CG, Soularue P, Gidrol X, Sentenac A, Werner M, Lefebvre O. Genome-wide location of yeast RNA polymerase III transcription machinery. EMBO J. 2003;22:4738-47.

50. Ronda C, Maury J, Jakociunas T, Jacobsen SAB, Germann SM, Harrison SJ, Borodina I, Keasling JD, Jensen MK, Nielsen AT. CrEdit: CRISPR mediated multi-loci gene integration in Saccharomyces cerevisiae. Microb Cell Fact. 2015;14:97.

51. Krappmann S, Sasse C, Braus GH. Gene targeting in Aspergillus fumigatus by homologous recombination is facilitated in a nonhomologous endjoining-deficient genetic background. Eukaryot Cell. 2006;5:212-5.

52. Ninomiya Y, Suzuki K, Ishii C, Inoue H. Highly efficient gene replacements in Neurospora strains deficient for nonhomologous end-joining. Proc Nat Acad Sci USA. 2004;101:12248-53.

53. Kooistra R, Hooykaas PJ, Steensma HY. Efficient gene targeting in Kluyveromyces lactis. Yeast. 2004;21:781-92.

54. Boulton SJ, Jackson SP. Saccharomyces cerevisiae Ku70 potentiates illegitimate DNA double-strand break repair and serves as a barrier to error-prone DNA repair pathways. EMBO J. 1996;15:5093-103.

55. Polotnianka RM, Li J, Lustig AJ. The yeast Ku heterodimer is essential for protection of the telomere against nucleolytic and recombinational activities. Curr Biol. 1998;8:831-4.

56. Laroche T, Martin SG, Gotta M, Gorham HC, Pryde FE, Louis EJ, Gasser SM. Mutation of yeast Ku genes disrupts the subnuclear organization of telomeres. Curr Biol. 1998:8:653-6.

57. Gravel S, Larrivee M, Labrecque P, Wellinger RJ. Yeast Ku as a regulator of chromosomal DNA end structure. Science. 1998:280:741-4.

58. Barnes G, Rio D. DNA double-strand-break sensitivity, DNA replication, and cell cycle arrest phenotypes of Ku-deficient Saccharomyces cerevisiae. Proc Natl Acad Sci USA. 1997;94:867-72.

59. Varzakas TH, Arvanitoyannis IS, Baltas H. The politics and science behind GMO acceptance. Crit Rev Food Sci Nutr. 2007;47:335-61.

60. Gibson B, Liti G. Saccharomyces pastorianus: genomic insights inspiring innovation for industry. Yeast. 2015;32:17-27.

61. Gorter de Vries AR, Pronk JT, Daran JG. Industrial relevance of chromosomal copy number variation in Saccharomyces yeasts. Appl Environ Microbiol. 2017;83:e03206-16.

62. Piatkowska EM, Naseeb S, Knight D, Delneri D. Chimeric protein complexes in hybrid species generate novel phenotypes. PLoS Genet. 2013:9:e1003836.

63. Nissim L, Perli SD, Fridkin A, Perez-Pinera P, Lu TK. Multiplexed and programmable regulation of gene networks with an integrated RNA and CRISPR/Cas toolkit in human cells. Mol Cell. 2014;54:698-710.

64. Nodvig CS, Nielsen JB, Kogle ME, Mortensen UH. A CRISPR-Cas9 system for genetic engineering of filamentous fungi. PLOS ONE. 2015:10:e0133085.

65. Weber J, Valiante V, Nodvig CS, Mattern DJ, Slotkowski RA, Mortensen UH, Brakhage AA. Functional reconstitution of a fungal natural product gene cluster by advanced genome editing. ACS Synth Biol. 2017;6:62-8.

66. Bolat I, Walsh MC, Turtoi M. Isolation and characterization of two new lager yeast strains from the WS34/70 population. Roum Biotechnol Lett. 2008;6:62-73

67. Nijkamp JF, van den Broek M, Datema E, de Kok S, Bosman L, Luttik MA Daran-Lapujade P, Vongsangnak W, Nielsen J, Heijne WHM, et al. De novo sequencing, assembly and analysis of the genome of the laboratory strain Saccharomyces cerevisiae CEN.PK113-7D, a model for modern industrial biotechnology. Microb Cell Fact. 2012;11:36.

68. Norrander J, Kempe T, Messing J. Construction of improved M13 vectors using oligodeoxynucleotide-directed mutagenesis. Gene. 1983;26:101-6.

69. Larkin MA, Blackshields G, Brown NP, Chenna R, McGettigan PA, McWilliam H, Valentin F, Wallace IM, Wilm A, Lopez R, et al. Clustal W and Clustal $X$ version 2.0. Bioinformatics. 2007:23:2947-8. 